\title{
Malignant Peritoneal Mesothelioma Interactome with 417 Novel Protein-Protein Interactions
}

Kalyani B. Karunakaran

Indian Institute of Science https://orcid.org/0000-0003-1279-7750

Madhavi K. Ganapathiraju ( $\square$ madhavi@pitt.edu )

University of Pittsburgh https://orcid.org/0000-0002-3825-0924

\section{Research Article}

Keywords: cancer biology, malignant mesothelioma, protein-protein interactions, systems biology, network analysis, drug repurposing, interactome

Posted Date: June 29th, 2021

DOI: https://doi.org/10.21203/rs.3.rs-663111/v1

License: (1) This work is licensed under a Creative Commons Attribution 4.0 International License.

Read Full License 


\title{
Malignant Peritoneal Mesothelioma Interactome with 417 Novel Protein-Protein Interactions
}

\author{
Kalyani B. Karunakaran ${ }^{1}$ and Madhavi K. Ganapathiraju ${ }^{2 *}$ \\ ${ }^{1}$ Supercomputer Education and Research Centre, Indian Institute of Science, Bengaluru, 560012, India \\ ${ }^{2}$ Department of Biomedical Informatics, School of Medicine, \\ Intelligent Systems Program, School of Computing and Information, \\ University of Pittsburgh, 5607 Baum Blvd, $5^{\text {th }}$ Floor, Pittsburgh, PA, 15206, USA
}

Corresponding author: madhavi@pitt.edu

\section{Abstract}

Malignant peritoneal mesothelioma (MPeM) is an aggressive cancer affecting the peritoneal lining of the abdominal cavity and intra-abdominal organs, with a median survival of $\sim 2.5$ years. We constructed an 'MPeM interactome' with over 400 computationally predicted protein-protein interactions (PPIs) and over 4,700 known PPIs of 59 literature-curated genes whose activity affects MPeM. Known PPIs of the 59 MPeM-associated genes were derived from BioGRID and HPRD databases. Novel PPIs were predicted by applying the HiPPIP algorithm, which computes features of protein pairs such as cellular localization, molecular function, biological process membership, genomic location of the gene, and gene expression in microarray experiments, and classifies the pairwise features as interacting or non-interacting based on a random forest model. $75.6 \%$ of the interactome and $65 \%$ of the novel interactors in it were supported by transcriptomic evidence in rodent and human peritoneal mesothelioma/mesothelial cell lines and tumor specimens. 152 drugs targeted 427 proteins in the MPeM interactome. Comparative transcriptome analysis of peritoneal mesothelioma-associated versus drug-induced gene expression profiles revealed 39 repurposable drugs, out of which 29 were effective against peritoneal/pleural mesothelioma and/or peritoneal metastasis/primary peritoneal cancer in clinical trials, animal models or cell lines. Functional modules of chromosomal segregation, transcriptional deregulation, positive regulation of IL-6 production and hematopoiesis were identified from the interactome. Genes with tissue-specific expression in 2 sites of extramedullary hematopoiesis (spleen and thymus) and those correlated with unfavorable prognosis in liver, renal, pancreatic and lung cancers were noted. MPeM interactome showed extensive overlap with the malignant pleural mesothelioma (MPM) interactome and MPM cell line expression profiles. Our findings demonstrate the utility of the MPeM interactome in discovering systems-level functional links among MPeM genes and generating clinically translatable results such as repurposed drugs.

Keywords: malignant mesothelioma, protein-protein interactions, systems biology, network analysis, drug repurposing

\section{Introduction}

Internal organs, such as the heart and lungs, and body cavities, such as the thoracic and abdominal cavities, are covered by a thin, slippery layer of cells called the "mesothelium". Mesothelioma is a rare but highly aggressive cancer that originates from this lining, is of types pericardial (heart), pleural (lung) and peritoneal (abdomen) mesothelioma; it is usually found in advanced stages and has a median survival of one year. ${ }^{1}$ Mesothelioma is intricately linked with exposure to asbestos but with a long latency period of few decades between exposure and the occurrence of the disease and does not have a non-invasive pre-malignant phase unlike other cancers. The focus of this work is on the genetics and biological mechanisms of MPeM. 
The peritoneum is a serosal membrane made up of two layers of mesothelial cells lining the abdominal cavity and intra-abdominal organs. MPeM affects this peritoneal lining and grows within the peritoneal space. ${ }^{1}$ Patients may exhibit symptoms such as weight loss, shortness of breath, chest and abdominal pain, increased abdominal girth and peritoneal effusion between the ages of 40-65 years. ${ }^{1}$ In contrast to pleural mesothelioma, where $80 \%$ of the cases are linked to asbestos exposure, only $8 \%$ of peritoneal mesothelioma cases have a history of asbestos exposure. $^{2}$ Factors predisposing patients to MPeM are less clear. ${ }^{2}$ MPeM appeared to associate with germline mutations more frequently than MPM. ${ }^{1}$ MPeM was more apparent among patients with a history of abdominal surgeries rather than asbestos exposure. ${ }^{1}$ In this case, chronic inflammation resulting from the procedure is presumed to have induced MPeM. Protracted inflammation of the peritoneum itself, or chronic peritonitis, seem to also pose risk. ${ }^{2}$ MPeM has a higher median survival rate than pleural mesothelioma (31 months versus 14 months), ${ }^{3}$ and is currently treated with a combination of pemetrexed and cisplatin, which elicits complete/partial responses in $26 \%$ (i.e. overall response rate) and disease stabilization (i.e. stable disease rate) in $45 \%$ of the patients. ${ }^{1}$

Several studies have speculated that the genetic factors driving peritoneal mesothelioma may be different from those driving pleural mesothelioma. One observation supporting this hypothesis is that 5p15 and $7 \mathrm{p} 12$ chromosomal gains were more common in peritoneal than in pleural mesothelioma. ${ }^{4}$ Multiple studies have examined the genetic underpinnings of MPeM. Hung et al. showed rearrangements in the gene ALK among a subset of MPeM patients having no asbestos fibres, history of therapeutic radiation and other genetic aberrations commonly found in MPeM tumors such as those in BAP1, SETD2 and NF2. ${ }^{5}$ Joseph et al. sequenced 510 cancerrelated genes in $13 \mathrm{MPeM}$ patients. ${ }^{6}$ Ugurluer et al. analyzed 236 somatic cancer-related mutations in $4 \mathrm{MPeM}$ patients. ${ }^{7}$ Chirac et al. performed comparative genomic hybridization (CGH) on samples collected from 33 French MPeM patients. ${ }^{8}$ Foster et al. performed DHPLC (denaturing high-performance liquid chromatography) and sequencing analysis on $29 \mathrm{MPeM}$ samples. ${ }^{9}$ Hung et al. examined molecular features of 26 diffuse malignant peritoneal mesothelioma using targeted next-generation sequencing. ${ }^{10}$ Pillai et al. investigated MUC1 expression and other prognostic factors in relation to survival in $42 \mathrm{MPeM}$ patients using immunohistochemistry. ${ }^{11}$ Varghese at al. performed gene expression analyses on tumor samples collected from 41 MPeM patients who underwent surgical cytoreduction and received regional intraoperative chemotherapy perfusion. ${ }^{12}$ Zaffaroni et al. investigated the expression of survivin and the effects of survivin knockdown in $32 \mathrm{MPeM}$ surgical specimens and an established MPM cell line respectively. ${ }^{13}$ The next step to discovering biological mechanisms is to understand how these genes play a role in the disease.

Protein-protein interactions (PPIs) drive the biological processes in cells including signal transduction, formation of cellular structures and enzymatic complexes. The molecular mechanisms of disease are often revealed by the PPIs of disease-associated genes. For example, the involvement of transcriptional deregulation in pleural mesothelioma pathogenesis was identified through mutations detected in BAP1 and its interactions with proteins such as HCF1, ASXL1, ASXL2, ANKRD1, FOXK1 and FOXK2. ${ }^{14}$ PPI of BAP1 with BRCA1 was central to understanding the role of BAP1 in growth-control pathways and cancer; BAP1 was suggested to play a role in BRCA1 stabilization. ${ }^{15,16}$ Studies on BAP1 and BRCA1 later led to clinical trials of the drug vinorelbine as a second line therapy for MPM patients, and the drug was shown to have rare or moderate effects in MPM patients. $^{17,18}$

Despite being critical to unravelling novel disease mechanisms and drugs, $\sim 75 \%$ of estimated PPIs are currently unknown and several disease-associated genes have no known PPIs. More than 600,000 PPIs are said to exist in the human interactome ${ }^{19}$ and only $\sim 150,000$ PPIs are known from PPI repositories such as HPRD ${ }^{20}$ and BioGRID $^{21}$. Detecting the PPIs using experimental techniques such as co-immunoprecipitation $(\mathrm{Co}-\mathrm{IP})^{22,23}$ is prohibitively laborious and time-consuming at large scale. Tens of thousands of PPIs are being added into the interactome through systematic high throughput studies with yeast two hybrid (Y2H) system ${ }^{24}$ and $\mathrm{AP}^{-\mathrm{MS}^{25}}$. Despite this, a large part of the interactome remains unknown. We have previously developed a computational 
model called HiPPIP (high-precision protein-protein interaction prediction) that was deemed highly accurate by computational evaluations, and experimental validations of 18 predicted PPIs, where all the tested pairs were shown to be true PPIs. ${ }^{26,27}$ We gained several high-impact biological insights from systems-level analysis of the interactome of disease-specific proteins which also included predicted PPIs. We published 2,156 novel PPIs related to MPM, schizophrenia, rheumatoid arthritis and congenital heart disease ${ }^{26,28-30}$. We constructed the MPM interactome from which we experimentally validated 5 novel PPIs of MPM-associated genes and identified 5 repurposable drugs using comparative transcriptome analysis; biological validity of the interactome was shown by the fact that more than $85 \%$ of this interactome was supported by genetic variant, transcriptomic and proteomic evidence related to MPM. ${ }^{28}$ Our analysis of the schizophrenia interactome resolved the apparent disconnect between schizophrenia GWAS genes and risk genes discovered in the pre-GWAS era, by showing that they belong to common pathways. ${ }^{26}$ We showed interactome overlap between schizophrenia and rheumatoid arthritis that may explain their shared pathology and inverse epidemiological relationship, ${ }^{29}$ a link between cilia, neuronal function and neurological disorders, ${ }^{31}$ the central role of cilia in congenital heart disease ${ }^{30}$ and the role of mitochondria in hypoplastic left heart syndrome. ${ }^{32}$ We were able to shortlist 12 repurposable drugs based on an integrated computational analysis of the schizophrenia drug-protein interactome; ${ }^{33}$ two of these drugs are in clinical trials as adjunctive therapy for schizophrenia (ClinicalTrials.gov NCT04887792; NCT03794076). Most recently we showed how the genes associated with malignant pleural mesothelioma identified by various high throughput studies were functionally linked within the mechanistic framework of the protein interactome. ${ }^{34}$

In this work, we constructed the 'MPeM interactome' by assembling the known and computationally predicted PPIs of the genes associated with malignant peritoneal mesothelioma, and studying pathways and biological processes associated with it. We analysed this interactome in the context of peritoneal mesothelioma transcriptomic datasets, tissue specificity of the constituent genes and their prognostic significance in other related cancers. We extended the MPeM interactome to include the drugs that target any of its proteins and analyzed it to identify a shortlist of 29 drugs that can potentially be repurposed for MPeM.

\section{Results}

We compiled a list of 59 genes associated with malignant peritoneal mesothelioma (MPeM) from nine studies; ${ }^{5-}$ 13 these genes harboured mutations, copy number aberrations, rearrangements or showed expression correlated with poor prognosis in MPeM patients or reduced cell survival or less favorable response to drugs in MPeM surgical specimens (Supplementary File 1). PPIs of these genes were collected from HPRD ${ }^{20}$ (Human Protein Reference Database) and BioGRID ${ }^{21}$ (Biological General Repository for Interaction Datasets). The HiPPIP algorithm described in our earlier work was applied to MPeM genes to discover hitherto unkown PPIs. ${ }^{35}$ HiPPIP computes features of protein pairs such as cellular localization, molecular function, biological process membership, genomic location of the gene, and gene expression in microarray experiments, and classifies the pairwise features as interacting or non-interacting based on a random forest model. ${ }^{26}$ The 'MPeM interactome' assembled in this manner contained 4,747 known PPIs and 417 novel PPIs connecting 58 MPeM-associated genes to 2,747 known interactors and 306 novel interactors (Fig. 1 and Supplementary File 2). The 59 ${ }^{\text {th }}$ MPeMassociated gene ADAM3A had neither known nor novel PPIs.

The number of known and computationally predicted novel PPIs for each of the MPeM genes are shown in Fig. 2 and Table 1; the novel interactors are listed in Table 1. 13 genes had 10 or less interactions each and 73 novel PPIs were predicted for all of the genes combined. There are 21 hub-genes that had more than 75 known PPIs each and 160 novel PPIs were predicted for all of the genes combined. 
Table 1. Novel PPIs of MPeM associated genes. Number of known PPIs ('K') and novel PPIs ('N')

\begin{tabular}{|c|c|c|c|}
\hline Gene & $\mathbf{K}$ & $\mathbf{N}$ & Novel Interactors \\
\hline \multicolumn{4}{|l|}{ ADAM3A } \\
\hline ALK & 16 & 12 & BIRC6, CLNS1A, DLG2, DNMT3A, HADHB, HMGB1, MSH2, NLRC4, RASA1, SMC1A, TSPAN16, TTC19 \\
\hline $\mathrm{AR}$ & 265 & & \\
\hline ARHGAP22 & 7 & 5 & ALG11, GPRIN2, MAPK8, PTPN20, ZNF488 \\
\hline ARID1A & 17 & 7 & CDC123, EDEM1, GMEB1, SMPDL3B, TAF12, THEMIS2, VPS41 \\
\hline ARID1B & 7 & 8 & ARMT1, GINM1, MAU2, MTHFD1L, NCOA6, PLEKHG1, TNFRSF19, ZBTB2 \\
\hline ASXL1 & 6 & 9 & DEFB115, DEFB116, FASN, ID1, IRAG1, MRPS7, NCOA6, POLD1, SRM \\
\hline BAP1 & 27 & 2 & PARP3, PLN \\
\hline BIRC5 & 25 & 8 & AANAT, AKAP6, CSNK1D, FASN, GCGR, NNMT, SSH2, TLE3 \\
\hline BRIP1 & 10 & 4 & HPN, MRPS23, PATZ1, PECAM1 \\
\hline $\mathrm{CDH} 5$ & 21 & 7 & CA7, CDH1, CDH16, CDH3, NQO1, PYGB, RND3 \\
\hline CDK12 & 9 & 9 & AP1M2, CDKN1A, FNDC8, GSDMB, PCDHB14, PCNT, PDLIM7, RPL13, SLFN12 \\
\hline CDKN2A & 168 & 5 & CA9, DNAI1, GLIPR2, NFX1, SIT1 \\
\hline CHEK2 & 80 & 5 & CRKL, FUS, GRK3, MN1, SUSD2 \\
\hline CTNNB1 & 187 & 11 & ARHGEF4, ASGR2, CCR1, CDC25A, CDK5, GLUD1, GNAI2, LAMB2, PTPRB, SSTR3, TJP1 \\
\hline DAXX & 113 & 10 & ADGRF3, CDSN, COL11A2, DXO, FBXO25, GNL1, GTF2H4, H2BC15, IKBKE, NRG2 \\
\hline DDX3X & 92 & 4 & CETN2, GABRE, RABEP1, TBC1D25 \\
\hline DPYD & 3 & 8 & CPB2, CSDE1, HPCA, KCNJ4, OVGP1, RPS6KA3, SRC, SULT2A1 \\
\hline EGFR & 409 & 4 & HEBP1, PHKG1, POM121L12, STAG3L4 \\
\hline EPHB1 & 13 & 10 & AADAC, ACP3, AMOTL2, CTPS1, GM2A, GTF2E1, MBD4, MRPL3, PCCB, UQCRC2 \\
\hline ESR1 & 365 & 6 & DDX43, FNDC1, KATNA1, RPL27A, SPDEF, UST \\
\hline FGF6 & 5 & 2 & CELSR1, KLRC2 \\
\hline HRAS & 146 & 7 & C6orf62, CCL4, HBG1, IGF2, INS, KCNQ1, ZFP36L2 \\
\hline IGKC & 4 & 2 & PLGLB2, REG3A \\
\hline IRS2 & 40 & 10 & CDKL1, CUL4A, EDEM1, LAMP1, MPO, NRAS, PROZ, PTPRR, RAP2A, SLC35B1 \\
\hline JAK1 & 72 & 8 & ANKRD13C, CBR1, DAB1, DHX9, NFYC, P2RY1, P3H1, VNN2 \\
\hline JUN & 180 & 9 & DAB1, GART, GPR18, ISCU, NFYC, PML, ROR1, SNIP1, TACSTD2 \\
\hline KDM6A & 11 & 7 & AKAP4, BMP15, ELK1, HSD17B10, MAGED2, RBM3, ZNF157 \\
\hline KDR & 60 & 8 & ALB, CACNA1S, CHIC2, GSTA2, KIT, SHOX2, SRP72, UTP3 \\
\hline KEAP1 & 77 & 15 & $\begin{array}{l}\text { ADH5, ARPC1B, BNIP3L, CARM1, ERBIN, LONP1, PANX1, RTN4R, S1PR2, SDC1, SENP1, SLC5A5, VAC14, ZNF177, } \\
\text { ZNF266 }\end{array}$ \\
\hline KMT2A & 31 & 13 & CKMT1A, DCTN1, EXOSC5, IL10RA, INPPL1, LAYN, MCTS1, PAFAH1B2, PLAAT4, PLPP2, RDX, TGFB2, VWA5A \\
\hline MAPK8 & 137 & 8 & ANXA8L1, ARHGAP22, CDC42, GDF10, GPRIN2, MT-CO1, PTPN20, TIMM23B \\
\hline MET & 112 & 12 & CAMSAP3, CAV1, FOXA3, KCND2, KLK2, MRPL17, PABPC1, RASSF7, SH3KBP1, SLC26A3, SLC26A4, SND1-IT1 \\
\hline MRE11 & 20 & 8 & -, CXCR5, DYNC1H1, ENDOD1, GPR83, JRKL, MLF2, TMEM126A \\
\hline MTOR & 52 & 7 & CA6, MECR, NPPB, PIK3CD, SLC2A5, SLC45A1, SLC9A1 \\
\hline MUC1 & 134 & 12 & ADGRL2, E2F2, GALE, IKBKE, LTB4R2, OAZ1, PKLR, PSMB4, RFX5, RPS24, SYT13, TFB2M \\
\hline NF2 & 74 & 4 & DRG1, LIF, OSM, PCNA \\
\hline NR2F2 & 18 & 3 & CHD2, RLBP1, SYCP3 \\
\hline NSD1 & 36 & 5 & NPR3, PRKCD, RING1, RTN4, SNRPF \\
\hline PBRM1 & 11 & 11 & AMIGO3, B4GALT7, CCRL2, CELSR3, FBXW8, FDX1, HSD17B7, PCBP4, RFX3, SS18L2, TMIE \\
\hline PIK3CA & 70 & 8 & ACOT8, ALCAM, CRIP1, LYPLA1, MAP3K9, PRKCI, RHOC, TRA2B \\
\hline PRDM1 & 10 & 6 & EXOSC3, FRK, HPGD, MAD2L1BP, MAN1A1, UTP18 \\
\hline PTEN & 356 & 9 & AKT3, ARL3, COL18A1, FGF7, KIF20B, LIPF, NCSTN, NR3C1, RBM15B \\
\hline RASSF1 & 70 & 5 & BANF1, LARS2, PFDN5, TMEM115, TMEM158 \\
\hline RB1 & 190 & 9 & CDADC1, CNTN3, COX17, CRYBA4, CSK, ESD, LRCH1, MTRF1, PCDHB5 \\
\hline RHEB & 29 & 6 & CENPE, MAPK15, NOS3, PIGB, PPP1R3C, PTPRN2 \\
\hline RICTOR & 11 & 8 & ANXA10, C5orf51, CTSW, IFT20, MROH2B, PHKB, PLCXD3, SIAH1 \\
\hline SDHB & 6 & 9 & CA6, FLOT1, MFAP2, RAP1GAP, RPL11, SLC45A1, SLC9A1, STMN1, TARBP1 \\
\hline SETD2 & 8 & 6 & EXOSC7, GMPPB, KLHL18, NDUFAF3, TEX264, TMA7 \\
\hline SMARCB1 & 112 & 3 & CNTNAP3, MYO18B, VPREB3 \\
\hline STK11 & 134 & 5 & FAM98B, MADCAM1, PPP6R1, SH3GL1, ZNF195 \\
\hline SUZ12 & 17 & 9 & ARHGAP5, CDK5R1, KLRC1, NOL4, NR1D1, PLA2G2A, RBBP4, SLC2A2, TBC1D29P \\
\hline TERT & 80 & 9 & ATPAF2, EXOC3, HMGB2, ICE1, IFT57, IRS1, NDUFS6, PDCD6, PTMA \\
\hline TP53 & 489 & 6 & CARD14, CFHR3, MMP10, POLR2A, RCVRN, ZNF367 \\
\hline TRAF7 & 12 & 6 & BTBD1, HAGHL, HDGF, NUDT16L1, PBXIP1, PPIC \\
\hline TRIO & 13 & 5 & DAP, DNAH5, MARCHF6, NIPBL, PRMT7 \\
\hline TSC1 & 99 & 11 & BRD3, CAPZA2, FCN1, FOLR2, NDUFA9, PAEP, PTPA, SLC16A6, TRAF2, TUBB4B, ZNF79 \\
\hline VEGFB & 6 & 5 & FOSL1, SF1, TFB1M, UCP2, UCP3 \\
\hline WT1 & 64 & 8 & B3GNT3, BST2, CALML5, CAPRIN1, FJX1, HIPK3, PAX6, PEX3 \\
\hline
\end{tabular}




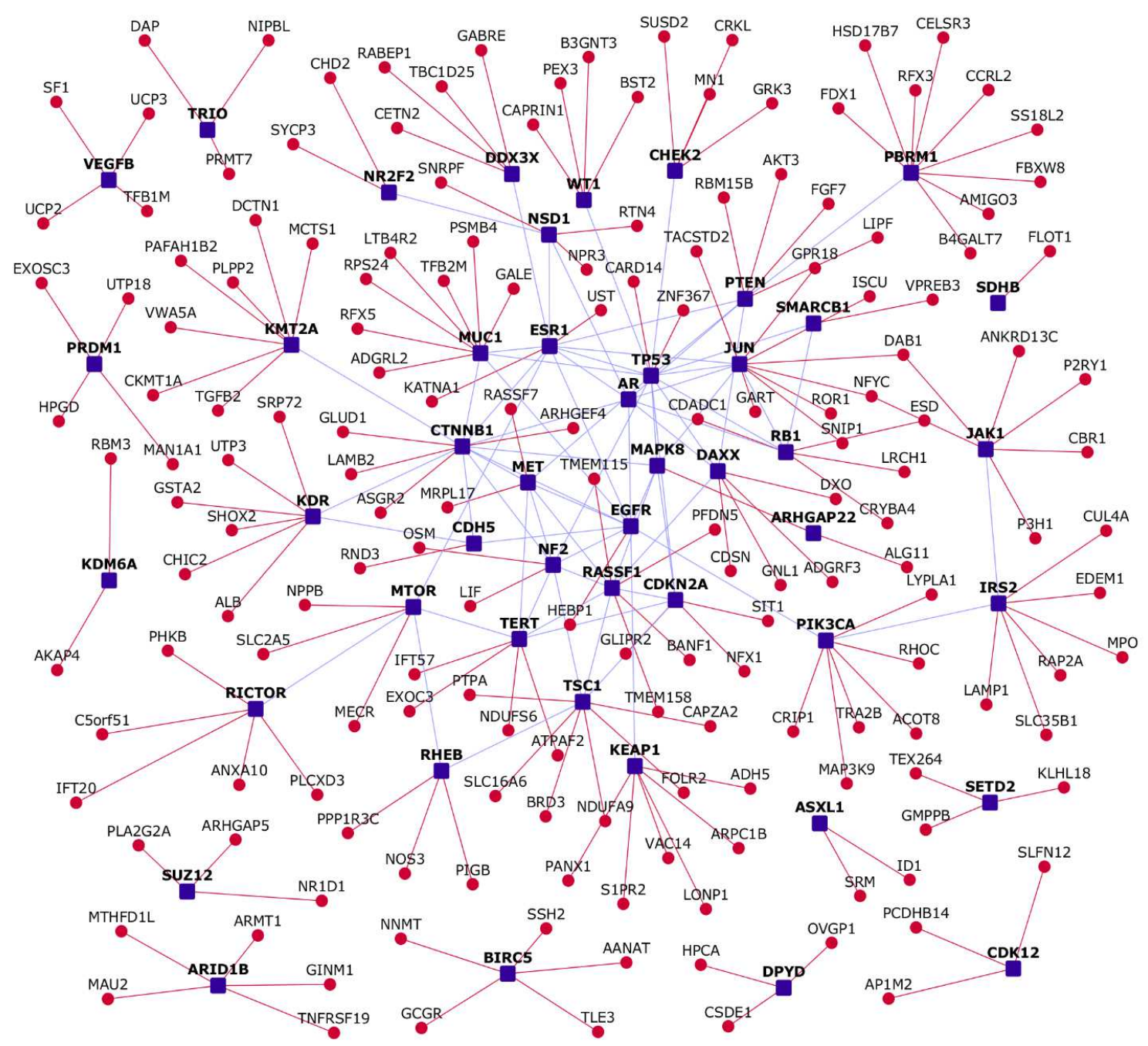

Figure 1. A partial network view of protein-protein interactions (PPIs) in the malignant peritoneal mesothelioma (MPeM) interactome: Genes are shown as nodes and PPIs as edges. As the complete MPeM network is very large, only a partial view showing a large connected component of MPeM candidate genes and their novel interactors, all of which have MPeM-related transcriptomic evidence (Supplementary Table X), is shown. Legend: dark blue square-shaped nodes: MPeM candidate genes; red nodes/edges: novel interactors/interactions; light blue nodes and blue edges: known interactors/interactions.

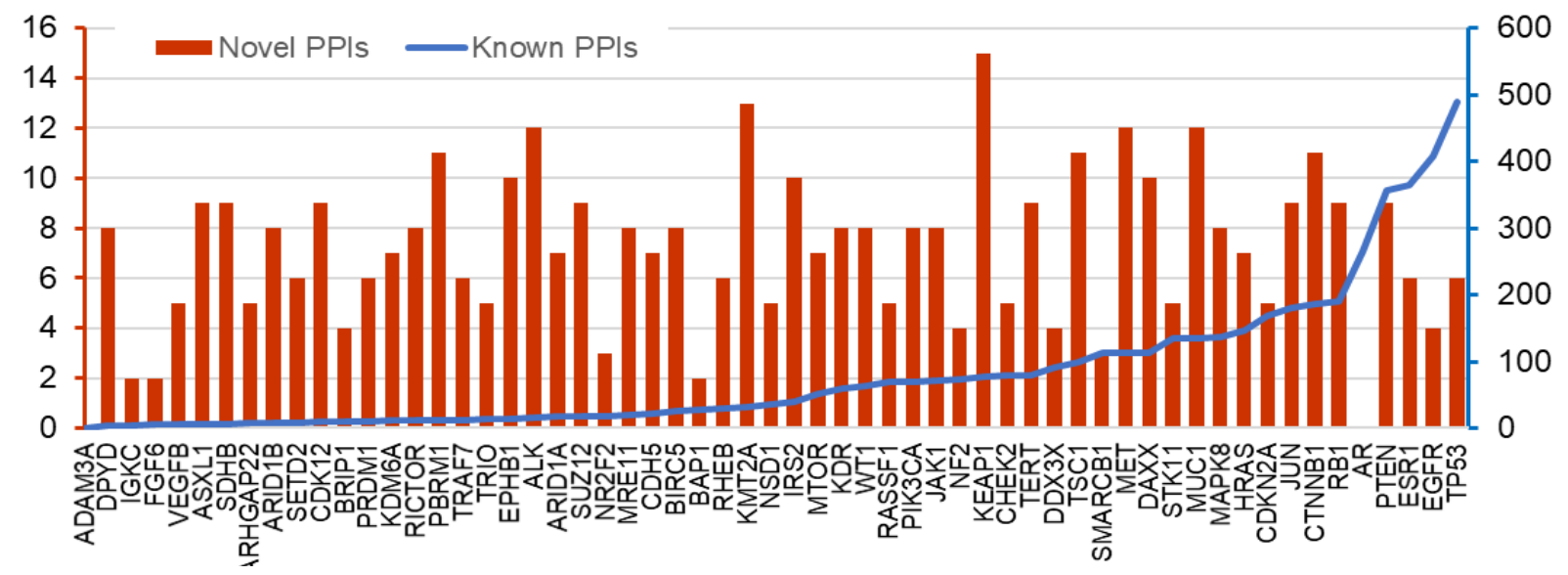

Figure 2. Number of Protein-Protein Interactions: The MPeM associated genes are listed along the $x$-axis, arranged in the ascending order of their number of known protein-protien interactions. The number of novel predicted PPIs and previously known PPIs are shown as red bars on primary axis (left) and blue line on secondary axis (right). For example. DPYD has three known PPIs and 8 novel PPIs, and AR has 265 known and zero novel PPIs. 
We used the HumanBase toolkit ${ }^{36}$ (https://hb.flatironinstitute.org/) to identify functional modules in the MPeM interactome. HumanBase employs shared k-nearest-neighbors and the Louvain community-finding algorithm to cluster the genes sharing the same network neighborhoods and similar Gene Ontology (GO) biological processes, into functional modules. 14 modules were detected of which 11 had more than 4 proteins each; 13 modules were enriched for the following biological processes: M1: chromosome segregation ( $Q$-value (false discovery rate adjusted p-value) <1E-08), M2: translation ( $Q$-value <1E-08), M3: hemopoiesis ( $Q$-value <1E-08), M4: covalent chromatin modification ( $Q$-value $<1 \mathrm{E}-08)$, M5, M9 and M10: transmembrane receptor protein tyrosine kinase signaling pathway ( $Q$-values of $<1 \mathrm{E}-08,2.82 \mathrm{E}-04$ and 2.64E-03 respectively), M6: histone modification ( $Q$-value <1E-08), M7: mRNA metabolic process ( $Q$-value <1E-08), M8: cell-cell adhesion ( $Q$-value = 2.86E-05), M11: negative regulation of intracellular signal transduction $(Q$-value $=3.98 \mathrm{E}-03)$, M12: negative regulation of hydrolase activity $(Q$-value $=5.84 \mathrm{E}-03)$, M13: cell-cell junction assembly $(Q$-value $=5.97 \mathrm{E}-03)$ and M14: positive regulation of interleukin-6 production $(Q$-value $=0.023)$. When novel interactors were not included, 9 modules of which all had 4 or more proteins each were detected. 52 novel interactors co-occurred with their corresponding MPeM-associated genes in these five modules: M3: IRS2-EDEM1, JUN-GPR18, JUN-PML, DPYD-SRC, ARID1B-PLEKHG1; M4: TRAF7-HDGF, TRAF7-PBXIP1, TRAF7-NUDT16L1, TRAF7HAGHL, DAXX-DXO, DAXX-GTF2H4, DAXX-GNL1, KEAP1-CARM1, KEAP1-LONP1, KEAP1-VAC14, ASXL1-SRM, ASXL1-FASN, ASXL1-MRPS7, MTOR-MECR, MTOR-SLC9A1, NSD1-RING1, NSD1PRKCD, RASSF1-TMEM115, RASSF1-BANF1, STK11-SH3GL1, STK11-PPP6R1, VEGFB-SF1, BAP1PARP3, NF2-DRG1; M5: MET-CAV1, KDR-KIT, KDR-SHOX2; M6: RICTOR-C5orf51, RICTOR-SIAH1, RICTOR-PHKB, TSC1-CAPZA2, PIK3CA-PRKCI, PIK3CA-ALCAM, KMT2A-RDX, PTEN-NR3C1, JAK1ANKRD13C, CDK12-GSDMB, PBRM1-HSD17B7; M7: SUZ12-RBBP4, WT1-CALML5, WT1-B3GNT3, WT1-PAX6, CDKN2A-DNAI1, CDKN2A-CA9, ALK-TSPAN16, EPHB1-AADAC and ESR1-SPDEF.

\section{Biological Process and Pathway Associations}

We identified the Reactome pathways enriched in the MPeM interactome using the gene set analysis toolkit called WebGestalt (Supplementary File 3). ${ }^{37}$ WebGestalt computes the statistical significance of association of the genes with a specific functional group (e.g. a Reactome Pathway) using Fischer's exact test and BenjaminiHochberg method for multiple test adjustment. The top-30 pathways associated with the MPeM are shown in Table 2. ${ }^{38}$

Table 2: Selected Pathways Associated with MPeM Interactome (P-value < 1E-15)

\begin{tabular}{|c|c|c|c|}
\hline Pathway & $\begin{array}{l}\text { Number } \\
\text { of genes }\end{array}$ & MPeM genes & Novel interactors \\
\hline Immune System & 578 & $\begin{array}{l}\text { BIRC5, CTNNB1, DDX3X, HRAS, IRS2, JAK1, } \\
\text { JUN, KEAP1, MAPK8, MRE11, MTOR, MUC1, } \\
\text { NF2, PIK3CA, PTEN, RICTOR, TP53, TRAF7 }\end{array}$ & $\begin{array}{l}\text { AKT3, AP1M2, ARPC1B, BST2, BTBD1, CALML5, } \\
\text { CAPZA2, CCL4, CENPE, CFHR3, CPB2, CRKL, } \\
\text { DCTN1, DEFB115, DEFB116, FBXW8, FCN1, GM2A, } \\
\text { GSTA2, KLRC1, KLRC2, LAMP1, LIF, MADCAM1, } \\
\text { MPO, NCSTN, NLRC4, NOS3, OSM, PAFAH1B2, } \\
\text { PANX1, PLA2G2A, PSMB4, PTPRN2, PYGB, } \\
\text { RAP1GAP, REG3A, SIAH1, SLC2A5 }\end{array}$ \\
\hline $\begin{array}{l}\text { Gene expression } \\
\text { (Transcription) }\end{array}$ & 517 & $\begin{array}{l}\text { AR, ARID1A, ARID1B, BIRC5, BRIP1, } \\
\text { CDK12, CDKN2A, CHEK2, CTNNB1, DAXX, } \\
\text { EGFR, ESR1, JUN, KMT2A, MET, MRE11, } \\
\text { MTOR, PBRM1, PRDM1, PTEN, RB1, RHEB, } \\
\text { RICTOR, SMARCB1, STK11, SUZ12, TP53, } \\
\text { TSC1 }\end{array}$ & $\begin{array}{l}\text { AKT3, DNMT3A, GTF2E1, ICE1, KIT, NLRC4, NR1D1, } \\
\text { PCBP4, PSMB4, SNRPF, TAF12, TFB2M, ZNF157, } \\
\text { ZNF195, ZNF266, ZNF79 }\end{array}$ \\
\hline
\end{tabular}




\begin{tabular}{|l|l|l|l|}
\hline $\begin{array}{l}\text { Developmental } \\
\text { Biology }\end{array}$ & 361 & $\begin{array}{l}\text { CTNNB1, EGFR, EPHB1, HRAS, IRS2, JUN, } \\
\text { KDM6A, MAPK8, MET, NR2F2, PIK3CA, } \\
\text { SUZ12, TRIO }\end{array}$ & $\begin{array}{l}\text { AKT3, ARPC1B, CACNA1S, CDSN, DAB1, FOXA3, } \\
\text { SIAH1, SLC2A2 }\end{array}$ \\
\hline $\begin{array}{l}\text { Cytokine Signaling } \\
\text { in Immune system }\end{array}$ & 269 & $\begin{array}{l}\text { BIRC5, IRS2, JAK1, JUN, MAPK8, MUC1, } \\
\text { PIK3CA, TP53 }\end{array}$ & BST2, CCL4, CRKL, GSTA2, LIF, OSM, PSMB4 \\
\hline Cell Cycle & 247 & $\begin{array}{l}\text { BIRC5, BRIP1, CDKN2A, CHEK2, MRE11, } \\
\text { RB1, TERT, TP53 }\end{array}$ & $\begin{array}{l}\text { AKT3, BANF1, CENPE, CETN2, DCTN1, MAU2, } \\
\text { NIPBL, PCBP4, PCNT, POLD1, PSMB4, SYCP3 }\end{array}$ \\
\hline $\begin{array}{l}\text { Cellular responses } \\
\text { to stress }\end{array}$ & 170 & $\begin{array}{l}\text { AR, CDKN2A, JUN, MAPK8, MRE11, MTOR, } \\
\text { RB1, SUZ12, TP53 }\end{array}$ & CAPZA2, DCTN1, ID1, PSMB4 \\
\hline $\begin{array}{l}\text { DNA Repair } \\
\text { family }\end{array}$ & 153 & BAP1, BRIP1, CHEK2, MAPK8, MRE11, TP53 & CETN2, CUL4A, MBD4, POLD1 \\
\hline $\begin{array}{l}\text { Deubiquitination } \\
\text { signaling cascades }\end{array}$ & 125 & AR, ASXL1, BAP1, ESR1, KEAP1, PTEN, TP53 & PSMB4 \\
\hline $\begin{array}{l}\text { SUMOylation } \\
\text { EGFR, FGF6, HRAS, IRS2, JAK1, JUN, MET }\end{array}$ & DLG2, FGF7, KIT, NRG2, PSMB4 \\
\hline
\end{tabular}

\section{Tissue-specificity of the genes in the MPeM interactome}

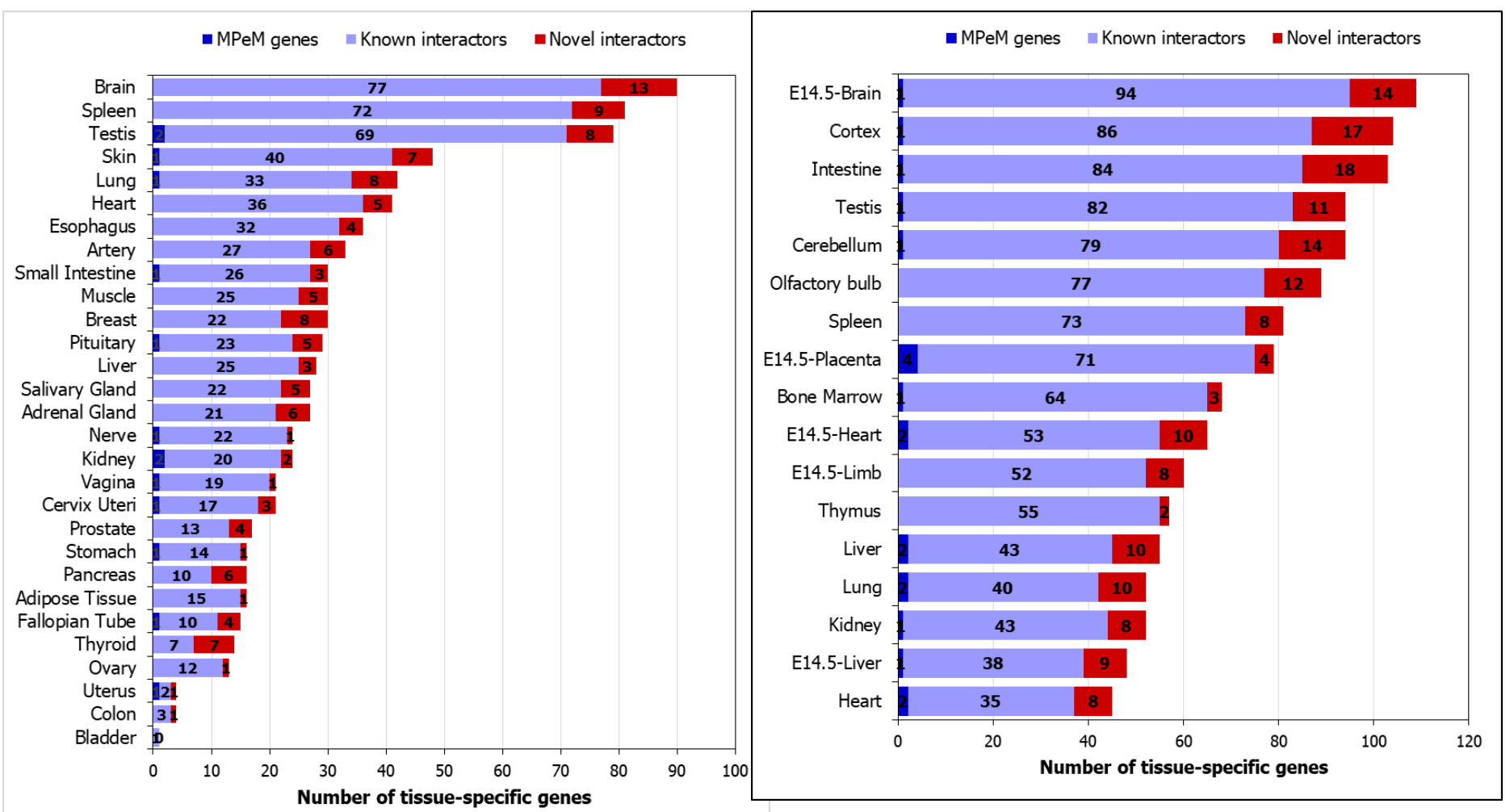

Figure 3. (A) Tissue-specificity of malignant peritoneal mesothelioma (MPeM) interactome genes in human organs: Tissue-specific expression of the genes in the interactome was examined using GTEx data. The graph shows the number of genes from the interactome that exhibit tissue specificity. The genes show at least 5-fold higher expression in a tissue ('tissue-enriched') or a group of 2-7 tissues compared to all the other tissues ('group-enriched'). (B) Tissue-specificity of malignant peritoneal mesothelioma (MPeM) interactome genes in mouse organs: Tissuespecific expression of the genes in the interactome was examined using mouse ENCODE data. The graph shows the number of genes from the interactome that exhibit tissue specificity. The genes show at least 5 -fold higher expression in a tissue ('tissue-enriched') or a group of 2-7 tissues compared to all the other tissues ('group-enriched'). 
We studied tissue specific expression of the proteins in the interactome using mouse ENCODE and GTEx data. ${ }^{39,40}$ Genes with an expression level greater than 1 TPM (transcripts per million) and relative expression at least 5-fold higher in a particular tissue (tissue-enriched) or a group of 2-7 tissues (group-enriched) were considered. ${ }^{41}$ Only two abdominal organs were found among the top-10 organs that showed enrichment in the interactome, namely, spleen and small intestine (Fig. 3A). This was contrary to the expectation that most of the genes in the interactome may show tissue specificity in abdominal organs lined by the peritoneum. Instead, the human organs that shared many genes with the interactome were brain, testis, skin, lung, heart, esophagus, artery and muscle (Fig. 3A). Similar specificity was observed in mouse organs/tissues such as intestine, cortex, cerebellum, olfactory bulb, testis and bone marrow, and embryonic tissues such as E14.5 brain, E14.5 placenta and E14.5 heart (Fig. 3B). The interactome showed statistical significance for enrichment in human orthologs of genes showing tissue-specific expression in mouse spleen (81 genes, $P$-value $=0.019$, odds ratio $=1.39)$ and thymus $(57$ genes, $P$-value $=0.028$, odds ratio $=1.42)($ Fig. 3B $) .10 \mathrm{MPeM}$-associated genes had novel PPIs with the orthologs of 10 spleen-specific mouse genes, namely, SMARCB1-VPREB3, JAK1-VNN2, RHEB-NOS3, ALK-NLRC4, IRS2-MPO, TSC1-FCN1, RICTOR-CTSW, HRAS-CCL4 and BIRC5-AANAT (MPeM genes are listed first in each pair) (i.e. 10 novel interactors had spleen-specificity).

We checked whether any of the genes in the interactome showed tissue-specific expression in the peritoneum using BaseSpace Correlation Engine. ${ }^{42,43}$ In this case, a gene was considered to be specific to a particular tissue, if the decrease in its expression in each of the other tissues relative to the tissue of interest is greater than 0.8 , among a list of tissues ranked by expression intensity (i.e. specificity index $>0.8$ ). By this definition, only 6 genes were considered to exhibit peritoneum-specific expression based on data from array-based gene expression studies. Nevertheless, OVGP1 which was predicted as a novel interactor of the MPeM-associated gene DPYD exhibited moderate specificity for peritoneum (specificity index $=0.57$ ).

\section{Overlap of the MPeM interactome with transcriptomic datasets}

198 out of $306(65 \%)$ novel interactors, and 2,353 (75.6\%) proteins overall of the MPeM interactome, showed differential gene expression in pre-clinical models and human tumor specimens of peritoneal mesothelioma (Supplementary File 4). These included human orthologs of genes that were differentially expressed in spontaneous or chemically induced peritoneal mesothelioma in rats, peritoneal mesothelioma cell lines cultured from mice exposed to crocidolite asbestos, or genes differentially expressed in MPeM tumor specimens, or human peritoneal mesothelial lines that were exposed to crocidolite. The interactome was significantly enriched in genes differentially expressed in granulocytic myeloid-derived suppressor cells (G-MDSCs) from spleens of mice bearing AB12 mesothelioma grafts versus naive neutrophils $(975$ genes, $P$-value $=2.02 \mathrm{E}-14$, odds ratio $=1.21)$, neutrophils infiltrating AB12 mesothelioma tumor grafts versus naive bone marrow derived neutrophils $(1,006$ genes, $P$-value $=1.97 \mathrm{E}-17$, odds ratio $=1.24)\left(\mathrm{GSE} 43254^{44}\right)$ and BCA induced peritoneal mesothelioma versus non-transformed mesothelial cell line (533 genes, $P$-value $=1.08 \mathrm{E}-04$, odds ratio $=1.15)$. A considerable number of genes in the interactome were differentially expressed in rat models of spontaneous and induced peritoneal mesothelioma, but their overlaps were not statistically significant. Specifically, 332 genes including 26 novel interactors were identified in o-Nitrotoluene (O-NT) induced peritoneal mesothelioma versus non-transformed mesothelial cell line (GSE4682 ${ }^{45}$ ), and 794 genes including 57 novel interactors in spontaneous malignant mesotheliomas from 2-year-old rats versus normal mesothelial Fred-PE cells (GSE47581 ${ }^{46}$ ). The interactome showed statistically significant overlaps with genes differentially expressed in two asbestos-exposed human peritoneal mesothelial cell lines, namely, LP9 mesothelial cells exposed for (i) 8 hours to $5 \mathrm{ug} / \mathrm{cm} 2 \mathrm{crocidolite}$ asbestos $\left(\mathrm{GSE} 14034^{47}: 303\right.$ genes, $P$-value $=6.39 \mathrm{E}-08$, odds ratio $=1.32$, GSE63966 ${ }^{48}: 560$ genes, $P$-value $=$ $1.24 \mathrm{E}-05$, odds ratio $=1.16$ ), and (ii) 8 hours to $1 \mathrm{ug} / \mathrm{cm} 2$ crocidolite asbestos $\left(\mathrm{GSE} 14034^{47}: 85\right.$ genes, $P$-value $=$ $8.76 \mathrm{E}-04$, odds ratio $=1.38$ ), versus untreated mesothelial cells, and primary peritoneal mesothelial HM3 cells exposed to $5 \mathrm{ug} / \mathrm{cm} 2$ crocidolite asbestos for 8 hours $\left(\mathrm{GSE} 63966^{48}: 797\right.$ genes, $P$-value $=3.99 \mathrm{E}-12$, odds ratio $=$ 1.22). 
In order to examine whether the interactome showed preferential enrichment for any specific subtype of peritoneal mesothelioma, we computed its overlap with genes found to be differentially expressed in biphasic versus epithelial peritoneal mesothelioma tumor specimens and vice versa. ${ }^{49}$ Significant enrichment was found with biphasic mesothelioma (118 genes, $P$-value $=2.17 \mathrm{E}-19$, odds ratio $=2.25)$, but not with epithelial mesothelioma (15 genes). This overlap included 4 genes predicted as novel interactors of 4 MPeM-associated genes (MPeM genes are shown in bold): ARID1A-TAF12, PIK3CA-LYPLA1, EPHB1-MRPL3 and KEAP1-LONP1. Out of the 15 genes in the interactome that showed specific expression in the epithelial subtype, 2 were predicted as novel interactors of MPeM-associated genes: VEGFB-SF1 and SUZ12-PLA2G2A.

Diffuse MPeM is known to share similar clinical presentation, morphology and immunostaining profiles with ovarian/primary peritoneal serous carcinoma (OC/PPC), and may hence be indistinguishable from the latter. ${ }^{50}$ Gene expression signatures characterizing these two tumors have been identified in an attempt to elucidate the molecular differences distinguishing them from one another. ${ }^{50}$ We computed the overlap of the MPeM interactome with these expression profiles. Out of the 12 genes in the interactome found to be differentially expressed in OC/PPC versus diffuse MPeM (including the MPeM-associated gene ESR1), 3 were predicted as novel interactors of MPeM-associated genes: HRAS-IGF2, JUN-TACSTD2 and CHEK2-SUSD2. 8 genes including the MPeM-associated gene KDR were found to be differentially expressed in diffuse MPeM versus OC/PPC.

A recent study had examined the gene expression profiles from the lungs of mice exposed to asbestos fibers (crocidolite and tremolite), an asbestiform fiber (erionite) and a mineral fiber (wollastonite). ${ }^{51}$ Crocidolite, tremolite and erionite are capable of inducing lung cancer and mesothelioma in human and animal models. ${ }^{51}$ On the other hand, wollastonite is a low pathogenicity fiber that shows no association with the incidence of lung cancer and mesothelioma in humans, or carcinogenesis in animal models. ${ }^{52}$ The MPeM interactome showed high statistical significance for genes differentially expressed upon exposure to crocidolite ( 322 genes, $P$-value $=3.5 \mathrm{E}-$ 13 , odds ratio $=1.44$ ) and marginal significance for genes differentially expressed upon wollastonite exposure (23 genes, $P$-value $=0.044$, odds ratio $=1.43)$. No enrichment was observed with erionite $(13$ genes $)$ and tremolite (64 genes).

In summary, these overlap studies (a) ascertained the biological validity of the MPeM interactome by showing that it contained genes that were biologically relevant to MPeM in the context of rodent xenograft/cell line mesothelioma models and human mesothelial cell lines, (b) highlighted genes that were specific to MPeM subtypes and those that help in distinguishing MPeM from other morphologically and histogenetically similar tumors, and (c) suggested a mechanistic framework to examine genes that appeared to be linked to MPeM etiology, based on transcriptomic evidence .

Disease associations We systematically analyzed the overlap of the MPeM interactome with prognostic genes of 20 cancer types. Data for correlation of gene expression and fraction of the patient population surviving after treatment for various cancer types were taken from Pathology Atlas. ${ }^{53}$ Genes with log-rank $P$-value $<0.001$ were considered to be prognostic. A positive correlation of high gene expression with reduced patient survival is considered unfavourable prognosis, whereas its correlation with increased patient survival is considered favourable. The interactome showed significant enrichment of genes associated with both prognosis for 6 cancer types, and either one type of prognosis for 10 other cancer types. The most significant enrichments were noted for unfavorable prognosis in liver cancer $(P$-value $=1.35 \mathrm{E}-25$, odds ratio $=1.43)$, renal cancer $(P$-value $=3.57 \mathrm{E}$ 18 , odds ratio $=1.31)$, pancreatic cancer $(P$-value $=4.18 \mathrm{E}-09$, odds ratio $=1.51)$ and lung cancer $(P$-value $=$ 4.01E-07, odds ratio $=1.62$ ). The most significant enrichments for favourable prognosis were noted in testis cancer $(P$-value $=7.7 \mathrm{E}-03$, odds ratio $=2.72)$, breast cancer $(P$-value $=8.23 \mathrm{E}-03$, odds ratio $=1.29)$, thyroid cancer $(P$-value $=0.013$, odds ratio $=1.41)$, and melanoma $(P$-value $=0.02$, odds ratio $=1.81)$. 
We studied the association of the interactome genes with diseases in the DisGeNET database. ${ }^{54}$ The top-5 diseases that were associated with MPeM were prostatic neoplasms (odds ratio $=1.98$ ), mammary neoplasms (odds ratio $=2.48$ ), stomach neoplasms (odds ratio $=2.41$ ), liver carcinoma (odds ratio $=2.34$ ) and lung neoplasms (odds ratio $=2.32$ ), all at $P$-value $<1 \mathrm{E}-15$. Several novel interactors were found to be associated with these diseases. For example, 13 novel interactors of MPeM-associated genes were associated with prostatic neoplasms (MPeM genes are shown in bold): MET-SLC26A4, DPYD-SULT2A1, CTNNB1-LAMB2, IRS2-MPO, HRASZFP36L2, VEGFB-UCP3, PRDM1-HPGD, NSD1-NPR3, KEAP1-SLC5A5, MET-FOXA3, RHEB-NOS3, HRAS-HBG1 and JAK1-CBR1. We employed Phenogrid from the MONARCH toolkit ${ }^{55}$ to identify the diseases that were most phenotypically similar to MPeM. The phenogrid algorithm identifies the common phenotypes between two given diseases. It then assesses the information content in each of these phenotypes (gene and disease associations) to assign a specific strength to the similarity observed between the diseases. Ovarian fibroma (OF), desmoplastic small round cell tumor (DSRCT), Budd-Chiari syndrome (BCS) and primary peritoneal carcinoma (PPC) are highly phenotypically like MPeM (similarity score > 80). We compiled 6, 43, 24 and 49 genes associated with OF, DSRCT, BCS and PPC respectively and checked their enrichment in the MPeM interactome. Statistically significant enrichment was shown by the genes associated with DSRCT $(P$-value $=4.16 \mathrm{E}-04$, odds ratio $=2.31)$ and PPC $(P$-value $=5.72 \mathrm{E}-08$, odds ratio $=2.98)$. Out of the 17 DSRCT-associated genes found in the MPeM interactome, 2 were MPeM-associated genes (WT1 and SMARCB1), and out of the 25 PPC-associated genes, 9 were MPeM-associated genes (DPYD, TP53, CHEK2, BRIP1, MRE11, PTEN, EGFR, KDR and ALK) and 2 were novel interactors (COL11A2 and ALB) of 2 MPeM-associated genes (DAXX and KDR).

Using MONARCH, ${ }^{55}$ we checked the enrichment of genes associated with 8 MPeM-associated phenotypes/clinical symptoms in the MPeM interactome, namely, ileus (intestinal obstruction preventing passage of intestinal contents), peritonitis (peritoneal inflammation), pedal edema (fluid accumulation in the feet and lower legs), ascites (fluid accumulation in the peritoneal cavity), dyspnea (labored breathing), abdominal pain, abdominal distention and abnormality of coagulation. The highest significance was shown by abdominal pain $(P-$ value $=1.23 \mathrm{E}-09$, odds ratio $=2.17)$, which was followed by pedal edema $(P$-value $=1.97 \mathrm{E}-07$, odds ratio $=$ $3.24)$, dyspnea $(P$-value $=1 \mathrm{E}-07$, odds ratio $=1.81)$, abdominal distention $(P$-value $=5.4 \mathrm{E}-05$, odds ratio $=2.04)$, abnormality of coagulation $(P$-value $=1.61 \mathrm{E}-03$, odds ratio $=1.89)$ and ascites $(P$-value $=0.01$, odds ratio $=1.65)$. Genes associated with ileus and peritonitis showed non-significant overlaps with the interactome; 3 out of the 16 ileus-associated genes and 2 out of 5 peritonitis-associated genes were found in the interactome. Overall, mutations in 14 novel interactors were found to be linked to these 8 disease symptoms (the MPeM-associated genes are shown in bold, and where marked with * showed the same phenotype association as their novel interactors): peritonitis, ascites and abdominal pain in humans: peritonitis, ascites and abdominal pain in humans: KEAP1-LONP1; dyspnea in humans: JUN-JUN, CTNNB1*-LAMB2, MAPK8-MT-CO1, EPHB1-MRPL3, KMT2A-TGFB2; abdominal pain in humans: KDR-KIT, ALK*-DNMT3A, BIRC5-GCGR; abdominal distention in humans: MET*-SLC26A4, SUZ12-SLC2A2, KEAP1-SLC5A5, MET*-SLC26A3; and, coagulation abnormality in humans: WT1-PEX3.

\section{Interconnections to Pleural Interactome}

We compared the overlap of MPeM interactome with the malignant pleural mesothelioma (MPM) interactome. ${ }^{\mathbf{3 4}}$ The two interactomes had 989 genes in common, which is a highly significant overlap $(P$-value $=3.18 \mathrm{E}-289$, odds ratio $=2.92$ ). This overlap included 4 core genes that were associated with MPM and MPeM (BAP1, CDKN2A, KDR and WT1), 29 PPIs between MPM and MPeM core genes, of which one is a novel PPI, 21 novel interactors of MPM and MPeM candidate genes, with the rest being known interactors. 38 MPM-associated genes, $41 \mathrm{MPeM}$ genes and the 4 genes common between them formed an intricately interconnected network of PPIs (Fig. 4). 6 of these are novel PPIs (FLT1-FLT3, TUBA1A-TUBA1C, RHGAP22-MAPK8, DPYD-SRC, JUNGART and TSC1-TUBB4B). 
Also, the MPeM interactome showed enrichment for several human MPM cell lines, including NCIH2052 $(P$ value $=1.45 \mathrm{E}-47$, odds ratio $=1.26), \mathrm{H} 2373 / \mathrm{PPMMill}(P$-value $=3.27 \mathrm{E}-29$, odds ratio $=1.35)$ and Hmeso $(P$ value $=4 \mathrm{E}-12$, odds ratio $=1.39)\left(\mathrm{GSE} 51447^{56}\right)$.

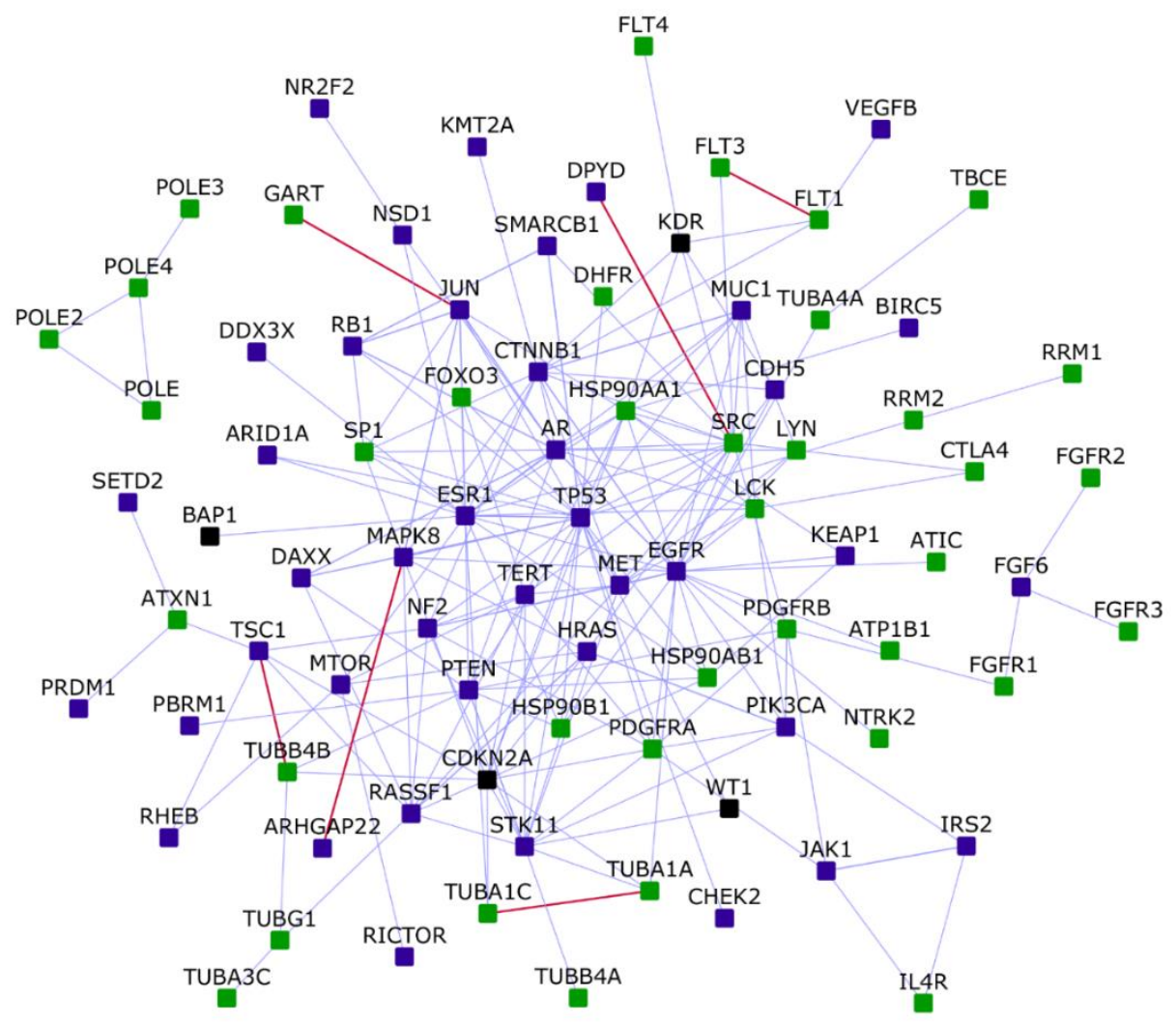

Figure 4. Interconnections of malignant peritoneal and pleural mesothelioma candidate genes: Square-shaped blue nodes: malignant peritoneal mesothelioma (MPeM) candidates, square-shaped green nodes: malignant pleural mesothelioma (MPM) candidates, square-shaped black nodes: genes that are MPeM as well as MPM candidates. Light blue and red colored edges indicate known and novel interactions respectively.

\section{Potentially repurposable drugs for malignant peritoneal mesothelioma}

We followed the established approach of comparing drug-induced versus disease-associated differential expression $^{57}$ to identify drugs for MPeM. For this, we used the software suite BaseSpace Correlation Engine (https://www.nextbio.com), ${ }^{42,43}$ which allows users to study the effect of diseases and/or drugs on thousands of pre-processed publicly available gene expression datasets and has helped to identify repurposable drug candidates for schizophrenia ${ }^{33}$ (currently undergoing clinical trials) and mesothelioma ${ }^{34}$ in the past. We constructed the MPeM drug-protein interactome that shows the drugs that target any protein in the MPeM interactome. 152 drugs were found to target 427 proteins in the MPeM interactome, including 16 MPeM-associated genes, 361 known interactors and 50 novel interactors. We selected 5 gene expression datasets associated with peritoneal mesothelioma, namely, granulocytic myeloid-derived suppressor cells (G-MDSCs) from spleens of mice bearing AB12 mesothelioma grafts versus naive neutrophils, neutrophils infiltrating AB12 mesothelioma tumor grafts versus naive bone marrow derived neutrophils (GSE43254 ${ }^{44}$ ), BCA induced peritoneal mesothelioma versus nontransformed mesothelial cell line, O-NT induced peritoneal mesothelioma versus non-transformed mesothelial cell line (GSE4682 ${ }^{45}$ ) and spontaneous malignant mesotheliomas from 2-year-old rats versus normal mesothelial Fred-PE cells (GSE47581 ${ }^{46}$ ). Then, we compiled a list of chemical compounds whose differential gene expression profiles (drug versus no drug) were negatively correlated with at least one of the 5 peritoneal mesothelioma differential gene expression datasets (disease versus control). Following this methodology, we identified 22 drugs 
negatively correlated with BCA induced peritoneal mesothelioma, 19 correlated with O-NT induced peritoneal mesothelioma, 24 correlated with spontaneous malignant mesothelioma, 5 correlated with G-MDSCs from the spleens of AB12 xenografts and 7 correlated with AB12 graft infiltrating neutrophils. Although in each case there were some genes that were differentially expressed in the same direction for both the drug and disorder, the overall effect on the entire transcriptome had an anti-correlation (Supplementary Files 5-9). We identified 39 drugs as potentially repurposable candidates for MPeM. Literature review supported the biological validity of 29 (74\%) out of these 39 drugs (Fig. 5), including 2 drugs (paclitaxel: NCT04000906 and imatinib: NCT00402766) that are already in clinical trials for MPeM. The evidence supporting these drugs is:

- Two drugs are part of the standard therapy for mesothelioma ${ }^{58}$ (pemetrexed and vinorelbine).

- One drug has shown activity against peritoneal mesothelioma, pleural mesothelioma and peritoneal metastasis (irinotecan). Although ineffective as a single agent, ${ }^{59}$ irinotecan elicited modest response rates and showed an acceptable toxicity profile in malignant pleural mesothelioma clinical trials, and produced an inhibitory effect on mesothelioma cell lines in combination with a p53 activator. ${ }^{60-62}$ Irinotecan was shown to be effective and tolerable in gastric cancer patients with peritoneal seeding. ${ }^{63}$ Importantly, irinotecan in combination with cisplatin showed efficacy and tolerability against peritoneal mesothelioma in a clinical setting. ${ }^{64}$

- Two drugs have shown activity against peritoneal mesothelioma in clinical settings and peritoneal metastasis in clinical trials/cell lines (paclitaxel and sirolimus). Paclitaxel appeared to be ineffective against MPM both as a single agent as well as in combination with other agents such as filgrastim and cisplatin. ${ }^{65-67}$ However, it has led to the complete remission of an MPeM patient for 20 months,${ }^{68}$ and clinical efficacy and an acceptable toxicity profile in ovarian, pancreatic and gastric cancers with peritoneal metastasis or serosal exposure. ${ }^{69,70}$ Sirolimus inhibited proliferation and increased cell death in MPM cell lines in combination with cisplatin, inhibited epithelial-to-mesenchymal transition in peritoneal mesothelial cell lines, and showed clinical efficacy in a patient with benign multicystic peritoneal mesothelioma. ${ }^{71-73}$

- Twelve drugs have shown activity against malignant pleural mesothelioma in clinical trials, animal models or cell lines (epirubicin, panobinostat, doxorubicin, imatinib, vinblastine, idarubicin, azacitidine, vorinostat, dactinomycin, acetylcysteine, staurosporine and quercetin). Epirubicin has shown a modest 10-20\% response rate in malignant mesothelioma patients. ${ }^{74}$ Panobinostat has been shown to have an inhibitory effect in mesothelioma cell lines and tumors in murine xenograft models. ${ }^{75}$ Doxorubicin has shown tolerable toxicity and improvement in the quality of life of MPM patients. ${ }^{76}$ Imatinib has shown both cytotoxicity and apoptosis in PDGFRB-positive mesothelioma cell lines, and has shown modest clinical efficacy in malignant mesothelioma patients. ${ }^{77,78}$ Vinblastine has shown improved progression-free survival rate and acceptable toxicity in combination with methotrexate and platinum. ${ }^{79-81}$ Both idarubicin and dactinomycin showed more cytotoxicity in MPM cell lines compared with pemetrexed and cisplatin. ${ }^{82}$ Azacitidine has shown inhibitory activity against malignant mesothelioma in clinical trials. ${ }^{83}$ Vorinostat induced apoptosis in mesothelioma cell lines. ${ }^{84}$ However, it did not provide any therapeutic benefit in patients with pleural mesothelioma. ${ }^{85}$ Acetylcysteine and quercetin have shown dose-dependent inhibition and time- and dosedependent inhibition in malignant mesothelioma cell lines respectively. ${ }^{86-88}$ Staurosporine was effective against mesothelioma tumors in murine xenograft models. ${ }^{89}$

- 6 drugs have shown activity against primary peritoneal cancer or peritoneal metastasis in other cancers (ruxolitinib, daunorubicin, dasatinib, topotecan, dexamethasone and nintedanib). The gene expression profile induced by ruxolitinib was shown to be negatively correlated with all the 5 peritoneal mesothelioma datasets. Ruxolitinib in combination with paclitaxel has been shown to inhibit tumor growth in a mouse model of advanced ovarian cancer with peritoneal metastasis. ${ }^{90}$ Daunorubicin induced side effects and showed no clinical activity against MPM. ${ }^{91}$ However, treatment with daunorubicin led to complete remission of a gastric Kaposi's sarcoma patient with peritoneal metastasis. ${ }^{92}$ Dasatinib showed inhibitory activity against peritoneal 
metastasis in a murine xenograft model of gastric cancer. ${ }^{93}$ Topotecan demonstrated clinical efficacy and tolerability in primary peritoneal carcinoma. ${ }^{94}$ Dexamethasone protected peritoneal mesothelial cells from epithelial-to-mesenchymal transition by acting on MAPK, GSK-3 $\beta$ and SNAI1 ${ }^{95}$ Nintedanib has been shown to inhibit peritoneal fibrosis in a mouse model by blocking mesothelial-to-mesenchymal transition. ${ }^{96}$

- 4 drugs have shown activity both against malignant pleural mesothelioma and peritoneal metastasis or sclerosis (methotrexate, resveratrol, everolimus and genistein). Methotrexate was shown to be clinically active against MPM in combination with gemcitabine, and against peritoneal metastasis in advanced gastric cancer. ${ }^{79,97-99}$ Everolimus induced AMPK/p38-mediated apoptosis in MPM cell lines and showed clinical activity in encapsulating peritoneal sclerosis. ${ }^{100-102}$ Resveratrol has produced an inhibitory effect on mesothelioma cell lines as a single agent as well as in combination with clofarabine, and has inhibited the adhesion of ovarian cancer cells to peritoneal mesothelial cells in vitro. ${ }^{26,103-105}$ Genisten has shown inhibitory activity against matrix metalloproteinases in malignant mesothelioma cell lines, and against peritoneal metastasis in intestinal adenocarcinomas in Wistar rats. ${ }^{106,107}$

- Two drugs have been shown to be effective in pleural/peritoneal effusions (mitoxantrone and vincristine) Mitoxantrone has shown only modest clinical activity in malignant mesothelioma. ${ }^{108}$ However, this drug has shown activity against pleural effusions in cancer patients. ${ }^{109}$ Vincristine was active against malignant peritoneal effusions in a murine xenograft model of Ehrlich ascites carcinoma. ${ }^{110}$

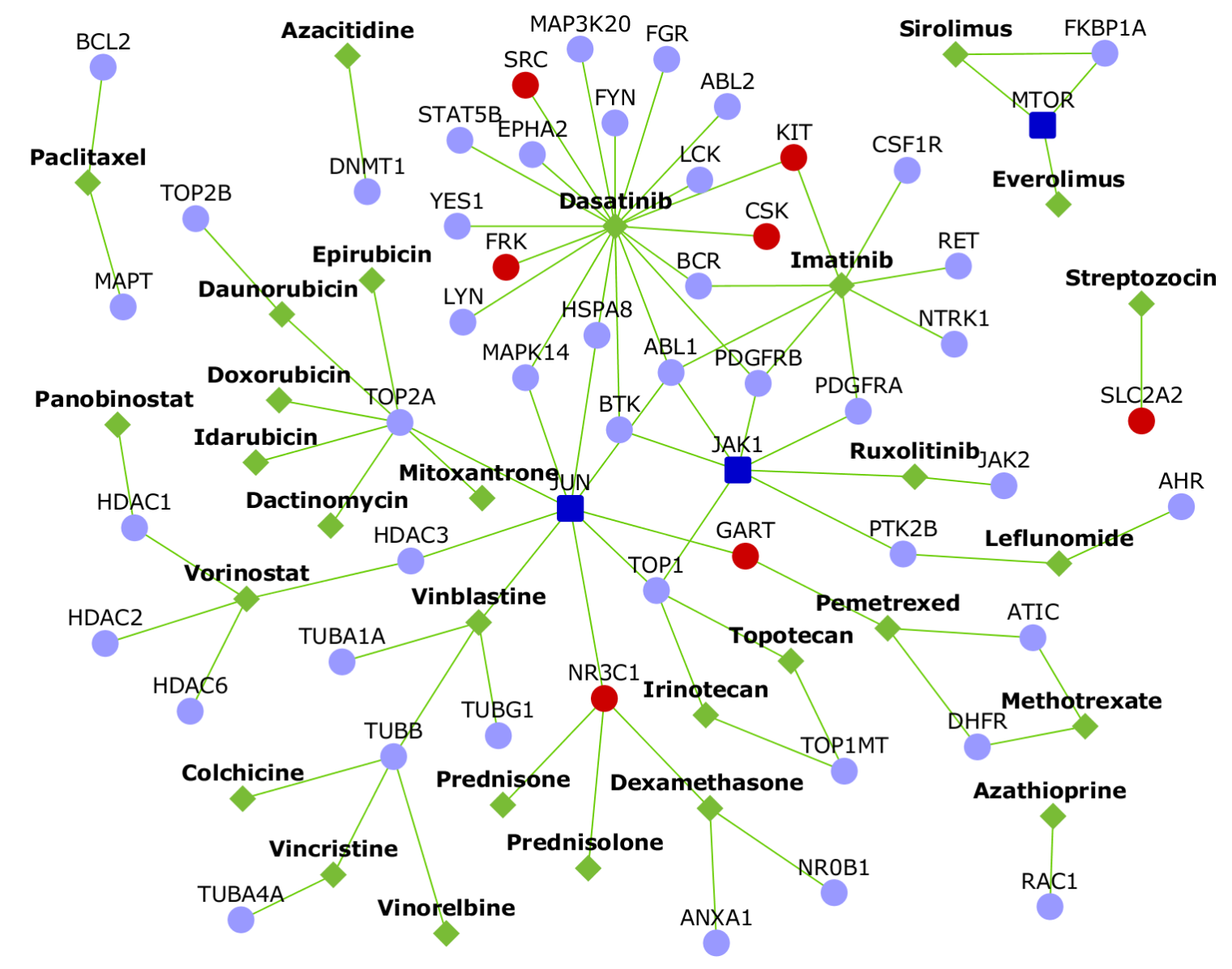

Figure 5. Repurposable drugs for malignant peritoneal mesothelioma (MPeM): The network shows 29 repurposable drugs (diamond-shaped green colored nodes) that target the proteins in the MPeM interactome. MPeM candidates are shown as dark blue nodes, their known interactors are light blue and novel interactors are red. 


\section{Discussion}

MPeM constitutes a substantial percentage (15\%-20\%) of all mesothelioma diagnoses and is additionally notable for its distinction from pleural mesothelioma, such as its limited association with asbestos exposure and proportionally higher prevalence in comparison with MPM among patients with germline mutations and without a history of asbestos exposure $\left(25 \%\right.$ versus $\left.7 \%{ }^{111}\right) .{ }^{1}$ In addition to this, three other factors that underscore the importance of MPeM-centric studies include the highly variable pattern of disease progression that it exhibits, risk for postoperative morbidity and mortality, and the increasing prevalence of peritoneal cases among mesothelioma patients without occupational exposure (given the current scenario in which the population of asbestos-exposed individuals is diminishing). ${ }^{1} 42 \%$ of MPeM patients survived for at least 5 years after cytoreductive surgery (CRS); however, instances of accelerated disease progression and death and survival for a long time with active disease have also been reported, indicating the variable nature of MPeM outcomes. ${ }^{1} \mathrm{~A}$ postoperative morbidity (complications and adverse events arising from the surgical procedure) of $35 \%$ and a mortality rate varying from $2 \%$ to $6 \%$ have been reported in patients who received CRS or hyperthermic intraoperative peritoneal chemotherapy (HIPEC), indicating the variable nature of surgical outcomes. ${ }^{1}$ Given the unique features of MPeM and its fatal nature, it is imperative that the molecular mechanisms underlying this disease are expeditiously discovered.

Although multiple studies have examined the genetic basis of MPeM, ${ }^{5-13}$ a mechanistic framework to interrogate its underlying cellular mechanisms and the functional consequence of the genes identified in these studies in an integrative manner is still lacking. In this study, we employed the protein interactome to discover the biological coherence of MPeM-associated genes identified in disparate studies, noting that MPeM may develop when PPIs are perturbed by genetic mutations or aberrant expression of MPeM-associated genes/proteins, ultimately leading to disrupted cellular functions. ${ }^{112}$ Extensive inter-connectivity and intra-connectivity of the network components in the human PPI network may augment the spreading of the effects of such perturbations to other proteins, encoded by genes that may/may not harbor any disease-associated alterations, through the network of their interactions, posing deeper implications for disease development, e.g. comorbidity, shared genetics and symptomatology among diseases and phenotypic responses to drugs. ${ }^{112}$ We constructed the MPeM interactome with more than 4,700 known interactions and more than 400 novel interactions of MPeM-associated genes collected from 9 studies, and conducted functional enrichment and transcriptome-based analyses to establish the biological validity of the interactome, gain valuable insights into MPeM etiology and identify drugs that may be repurposed to treat MPeM.

A key finding from our study was that more than $75 \%$ of the interactome as a whole, and more than $60 \%$ of the novel interactors that were predicted to interact with MPeM-associated genes by the HiPPIP algorithm, had MPeM-related transcriptomic aberrations in rodent and human cell line models. This demonstrates the validity of our interactome-based method due to two reasons: we (a) identified other genes that were previously identified in MPeM-related gene expression studies using an unbiased approach from the MPeM interactome, and (b) showed that they were closely connected to a curated set of MPeM-associated genes and that they could be functionally relevant to MPeM etiology in an interdependent manner. Another key finding was that more than $70 \%$ of the repurposable drugs that we identified using comparative transcriptome analysis has been shown to be effective against peritoneal mesothelioma, pleural mesothelioma, peritoneal metastasis and/or primary peritoneal cancer in clinical trials, animal models or cell lines. The drug-associated expression profiles analyzed in this study were induced in a wide variety cell lines. The effect of the proposed drugs should be specifically examined in human peritoneal mesothelioma cell lines or animal models.

Human orthologs of spleen-specific and thymus-specific mouse genes showed significant enrichment in the interactome, instead of major abdominal organs. This finding seemed to be in line with the identification of hematopoiesis as a functional module enriched in the MPeM interactome. Both spleen and thymus are 
hematopoietic sites regulating the proliferation, maturation and activation of lymphoid cells outside of the bone marrow (extramedullary hematopoiesis). Expansion of myeloid cells in the spleen through the process of extramedullary hematopoiesis resulting in splenomegaly has been observed in BAP1 knockout mice. ${ }^{113}$ Further investigations may be necessary to elucidate the functional consequences of this phenomenon in MPeM. 56\% (33) of the MPeM-associated genes that we had used for interactome construction were involved in chromosomal events such as copy number gain/loss, gene loss, deletion and gene rearrangement. In line with this, chromosome segregation was identified as the most enriched functional module in the interactome. We identified 10 novel interactors involved in chromosome segregation, out of which 7 were predicted to interact with MPeM-associated genes involved in chromosomal events (indicated with *; MPeM genes are shown in bold): RASSF1*-LARS2, ESR1-KATNA1，ARID1B*-MTHFD1L， RHEB*_CENPE，VEGFB*-TFB1M，TP53-ZNF367， ASXL1POLD1, JUN*_GART, PTEN*-KIF20B and KEAP1*-SENP1. Transcriptional deregulation seemed to be critical to MPeM etiology, as indicated by the identification of functional modules involved in covalent chromatin modification and translation; together, these modules contained 53 novel interactors of MPeM-associated genes. Elevated expression of an anti-apoptotic factor called survivin (BIRC5) induced by the cytokine IL-6 has been reported in MPeM patients; knockdown of this gene led to increased (spontaneous and drug-induced) apoptosis. ${ }^{13}$ In line with this, positive regulation of IL-6 production has been identified as an enriched functional module, which also includes 13 novel interactors of MPeM-associated genes: NR2F2-SYCP3, ESR1-DDX43, RB1LRCH1, RB1-PCDHB5, MRE11-GPR83, PBRM1-FBXW8, RB1-CDADC1, MET-FOXA3, RB1-CNTN3, SMARCB1-MYO18B, TRIO-DNAH5, ARHGAP22-ZNF488 and SDHB (and MTOR)-SLC45A1. Future studies could concentrate on validating the novel PPIs involved in these functional modules relevant to MPeM etiology and the extensive interconnections noted among MPeM-associated genes (of which only a portion has been shown in Fig. 1).

As noted earlier, a higher prevalence of MPeM compared to MPM has been observed among carriers of germline mutations. ${ }^{1}$ These mutations (e.g. in BAP1 and TP53) may predispose MPeM patients to multiple other cancers. ${ }^{1}$ We identified a significant enrichment of genes, whose elevated expression positively correlates with (a) unfavourable prognosis in liver, renal, pancreatic and lung cancers and (b) favourable prognosis in testis, breast, thyroid and skin cancers, in the MPeM interactome. Prolonged survival has been reported in mesothelioma patients with germline mutations, indicating that these mutations may sometimes mediate inhibition of aggressive tumor growth. ${ }^{1}$ Additionally, prolonged survival has been associated with the occurrence of other cancers in carriers of germline mutations. ${ }^{1}$ Genetic screening to identify aberrations in prognostic genes may guide clinicians in patient stratification (based on their survival time and risk of developing other cancers) and in devising better treatment strategies.

We showed extensive overlap of the MPeM interactome with the MPM interactome, interconnections between MPeM-associated genes and MPM-associated genes in the interactome and significant enrichment of the MPeM interactome in MPM cell lines. Further investigations focused on these findings may provide clues on the etiological diversification and common genetic underpinnings of MPeM and MPM.

In summary, our study provides a network-level view of MPeM-associated genes and their functional consequences. The MPeM interactome will serve as a functional landscape to integrate and analyse MPeM related multi-omics data and will inform and catalyze future investigations on the genetic basis of MPeM and biomedical studies seeking to improve clinical interventions in MPeM. 


\section{Methods}

\section{Compilation of MPeM-associated genes and prediction of novel interactions}

A list of $59 \mathrm{MPeM}$-associated genes that harboured mutations, copy number aberrations, rearrangements or showed expression correlated with poor prognosis in MPeM patients or reduced cell survival or less favorable response to drugs in MPeM surgical specimens was compiled from eight studies..$^{5-13}$ Novel PPIs of the proteins encoded by these genes were predicted using the HiPPIP model that we developed. ${ }^{35}$ Each MPeM protein (say N1) was paired with each of the other human proteins say, (M1, M2,..Mn), and each pair was evaluated with the HiPPIP model. ${ }^{35}$ The predicted interactions of each of the MPeM proteins were extracted (namely, the pairs whose score is $>0.5$, a threshold which through computational evaluations and experimental validations was revealed to indicate interacting partners with high confidence). The interactome figures were created using Cytoscape. ${ }^{114}$

\section{Identification of functional modules}

Functional gene modules were extracted using the HumanBase toolkit ${ }^{36}$ (https://hb.flatironinstitute.org/). HumanBase uses shared k-nearest-neighbors and the Louvain community-finding algorithm to cluster the genes sharing the same network neighborhoods and similar GO biological processes into functional modules. The pvalues of the terms enriched in the modules are calculated using Fisher's exact test and Benjamini-Hochberg method.

\section{Functional enrichment analysis}

Biological process $\left(\right.$ Gene Ontology ${ }^{115}$ ), pathway (Reactome ${ }^{116}$ ) and disease (DisGeNET ${ }^{54}$ ) enrichments were computed using WebGestalt. ${ }^{37}$ WebGestalt computes the distribution of genes belonging to a particular functional category in the input list and compares it with the background distribution of genes belonging to this functional category among all the genes that belongs to any functional category in the database selected by the user. Statistical significance of functional category enrichment is computed using Fisher's exact test, and corrected using the Benjamini-Hochberg method for multiple test adjustment. Annotations with FDR-corrected p-value < 0.05 were considered significant.

\section{Tissue-specific expression analysis}

Tissue-specificity of the genes in the MPeM interactome were checked using TissueEnrich. ${ }^{117}$ The analysis was based on tissue-specific genes compiled from GTEx and Mouse ENCODE. ${ }^{39,40}$ This included 'tissue-enriched genes' with at least 5-folds higher mRNA levels in a particular tissue compared to all the other tissues, 'groupenriched genes' with at least 5-folds higher mRNA levels in a group of 2-7 tissues and 'tissue-enhanced genes' with at least 5-folds higher mRNA levels in a particular tissue compared to average levels in all tissues.

\section{Network overlap analysis}

Statistical significance of the overlaps between genes in the MPeM and MPeM interactomes was computed based on hypergeometric distribution.

\section{Identification of prognostic cancer genes}

Data for correlation of gene expression and fraction of patient population surviving after treatment of 20 cancer types was taken from Pathology Atlas. ${ }^{53}$ Genes with $\log$-rank $P$-value $<0.001$ were considered to be prognostic. Unfavorable prognosis indicates positive correlation of high gene expression with reduced patient survival. 


\section{Identification of repurposable drugs}

The list of chemical compounds whose gene expression profiles correlated negatively with 5 gene expression datasets associated with peritoneal mesothelioma were compiled using the BaseSpace correlation software (https://www.nextbio.com) (List 1). The datasets considered were granulocytic myeloid-derived suppressor cells (G-MDSCs) from spleens of mice bearing AB12 mesothelioma grafts versus naive neutrophils, neutrophils infiltrating AB12 mesothelioma tumor grafts versus naive bone marrow derived neutrophils (GSE43254 ${ }^{44}$ ), BCA induced peritoneal mesothelioma versus non-transformed mesothelial cell line, O-NT induced peritoneal mesothelioma versus non-transformed mesothelial cell line $\left(\mathrm{GSE} 4682^{45}\right)$ and spontaneous malignant mesotheliomas from 2-year-old rats versus normal mesothelial Fred-PE cells (GSE47581 ${ }^{46}$ ). Next, we identified drugs that targeted at least one gene in in the MPeM interactome using Drug Bank. ${ }^{118}$ We then compared list 1 and list 2 to identify the drugs that not only target proteins in the interactome but are also negatively correlated with MPeM-associated gene expression profiles.

\section{Acknowledgements}

This work has been funded by Mesothelioma Applied Research Foundation (MARF), USA (612571, Principal Investigators: Dr. Waqas Amin and Prof. Madhavi K. Ganapathiraju) and in part by National Institute of Occupational Safety and Health (NIOSH), USA (U24OH009077, Principal Investigator: Prof. Michael J. Becich). The content is solely the responsibility of the authors and does not necessarily represent the official views of MARF or NIOSH. MKG thanks Prof. Michael Becich for support and for funding. MKG thanks Dr. Waqas Amin for joint effort in securing funding that supported this work. Authors thank Prof. N. Balakrishnan of Indian Institute of Science for supporting KBK.

\section{Author contributions}

MKG conceptualized and supervised the study and carried out interactome prediction and analysis. KBK carried out studies of overlap of the interactome with high-throughput data and literature-based evidence gathering. Manuscript has been written by KBK and edited by MKG. Manuscript has been read and approved by all authors.

\section{Competing interests}

None.

\section{Data and materials availability}

On journal/preprint archive.

\section{References}

1 Carbone, M. et al. Mesothelioma: scientific clues for prevention, diagnosis, and therapy. CA: a cancer journal for clinicians 69, 402-429 (2019).

Robinson, B. W. \& Lake, R. A. Advances in malignant mesothelioma. New England Journal of Medicine 353, 15911603 (2005).

Amin, W. et al. Factors influencing malignant mesothelioma survival: a retrospective review of the National Mesothelioma Virtual Bank cohort. F1000Research 7 (2018). 
Takeda, M. et al. Comparison of genomic abnormality in malignant mesothelioma by the site of origin. Journal of clinical pathology 67, 1038-1043 (2014).

Hung, Y. P. et al. Identification of ALK rearrangements in malignant peritoneal mesothelioma. JAMA oncology 4, 235-238 (2018).

Joseph, N. M. et al. Genomic profiling of malignant peritoneal mesothelioma reveals recurrent alterations in epigenetic regulatory genes BAP1, SETD2, and DDX3X. Modern Pathology 30, 246-254 (2017).

Ugurluer, G. et al. Genome-based mutational analysis by next generation sequencing in patients with malignant pleural and peritoneal mesothelioma. Anticancer research 36, 2331-2338 (2016).

Chirac, P. et al. Genomic copy number alterations in 33 malignant peritoneal mesothelioma analyzed by comparative genomic hybridization array. Human pathology 55, 72-82 (2016).

Foster, J. M. et al. Clinical implications of novel activating EGFR mutations in malignant peritoneal mesothelioma. World journal of surgical oncology 8, 88 (2010).

Hung, Y. P. et al. Molecular characterization of diffuse malignant peritoneal mesothelioma. Modern Pathology, 111 (2020).

Pillai, K., Pourgholami, M. H., Chua, T. C. \& Morris, D. L. MUC1 has prognostic significance in malignant peritoneal mesothelioma. The International journal of biological markers 28, 303-312 (2013).

12 Varghese, S. et al. Activation of the phosphoinositide-3-kinase and mammalian target of rapamycin signaling pathways are associated with shortened survival in patients with malignant peritoneal mesothelioma. Cancer 117, 361-371 (2011).

13 Zaffaroni, N. et al. Survivin is highly expressed and promotes cell survival in malignant peritoneal mesothelioma. Analytical Cellular Pathology 29, 453-466 (2007).

Bott, M. et al. The nuclear deubiquitinase BAP1 is commonly inactivated by somatic mutations and 3p21. 1 losses in malignant pleural mesothelioma. Nature genetics 43, 668 (2011).

15 Jensen, D. E. et al. BAP1: a novel ubiquitin hydrolase which binds to the BRCA1 RING finger and enhances BRCA1mediated cell growth suppression. Oncogene 16, 1097 (1998).

Hakiri, S. et al. Functional differences between wild-type and mutant-type BRCA1-associated protein 1 tumor suppressor against malignant mesothelioma cells. Cancer science 106, 990-999 (2015).

17 Zauderer, M. G. et al. Vinorelbine and gemcitabine as second-or third-line therapy for malignant pleural mesothelioma. Lung Cancer 84, 271-274 (2014).

18 Zucali, P. et al. Vinorelbine in pemetrexed-pretreated patients with malignant pleural mesothelioma. Lung Cancer 84, 265-270 (2014). level. Chemical reviews 116, 4884-4909 (2016). 

(2008).

21 Stark, C. et al. BioGRID: a general repository for interaction datasets. Nucleic acids research 34, D535-D539 (2006).

22 Blasche, S. \& Koegl, M. Analysis of Protein-Protein Interactions Using LUMIER Assays. Virus-Host Interactions: Methods and Protocols, 17-27 (2013).

23 Trepte, P. et al. DULIP: a dual luminescence-based co-immunoprecipitation assay for interactome mapping in mammalian cells. Journal of molecular biology 427, 3375-3388 (2015).

24 Luck, K. et al. A reference map of the human binary protein interactome. Nature, 1-7 (2020).

25 Huttlin, E. L. et al. Dual Proteome-scale Networks Reveal Cell-specific Remodeling of the Human Interactome. bioRxiv (2020).

26 Ganapathiraju, M. K. et al. Schizophrenia interactome with 504 novel protein-protein interactions. npj Schizophrenia 2, 16012 (2016).

27 Zhu, J. et al. Antiviral activity of human OASL protein is mediated by enhancing signaling of the RIG-I RNA sensor. Immunity 40, 936-948 (2014).

28 Karunakaran, K. B., Yanamala, N., Boyce, G., Becich, M. J. \& Ganapathiraju, M. K. Malignant Pleural Mesothelioma Interactome with 364 Novel Protein-Protein Interactions. Cancers 13, 1660 (2021).

29 Malavia, T. et al. Generating testable hypotheses for schizophrenia and rheumatoid arthritis pathogenesis by integrating epidemiological, genomic and protein interaction data npj Schizophrenia in Press (2017).

$30 \mathrm{Li}$, Y. et al. Global genetic analysis in mice unveils central role for cilia in congenital heart disease. Nature 521, 520524, doi:10.1038/nature14269 (2015).

31 Karunakaran, K. B., Balakrishnan, N. \& Ganapathiraju, M. K. Interactome of SARS-CoV-2/nCoV19 modulated host proteins with computationally predicted PPIs. Research Square (2020).

32 Liu, X. et al. The complex genetics of hypoplastic left heart syndrome. Nat Genet 49, 1152-1159, doi:10.1038/ng.3870 (2017).

33 Karunakaran, K. B., Chaparala, S. \& Ganapathiraju, M. K. Potentially repurposable drugs for schizophrenia identified from its interactome. Scientific Reports 9, 12682, doi:10.1038/s41598-019-48307-w (2019).

34 Karunakaran, K. B., Yanamala, N., Boyce, G. \& Ganapathiraju, M. K. Mesothelioma Interactome with 367 Novel Protein-Protein Interactions. bioRxiv, 459065 (2018).

35 Ganapathiraju, M. K., Karunakaran, K. B. \& Correa-Menendez, J. Predicted protein interactions of IFITMs may shed light on mechanisms of Zika virus-induced microcephaly and host invasion. F1000Res 5, 1919, doi:10.12688/f1000research.9364.2 (2016).

Krishnan, A. et al. Genome-wide prediction and functional characterization of the genetic basis of autism spectrum disorder. Nature neuroscience 19, 1454-1462 (2016). 
Liao, Y., Wang, J., Jaehnig, E. J., Shi, Z. \& Zhang, B. WebGestalt 2019: gene set analysis toolkit with revamped Uls and APIs. Nucleic acids research (2019).

38 Liu, Y. et al. Global prevalence of congenital heart disease in school-age children: a meta-analysis and systematic review. BMC cardiovascular disorders 20, 1-10 (2020).

39 Lonsdale, J. et al. The Genotype-Tissue Expression (GTEx) project. Nature Genetics 45, 580-585, doi:10.1038/ng.2653 (2013).

40 Davis, C. A. et al. The Encyclopedia of DNA elements (ENCODE): data portal update. Nucleic acids research 46, D794-D801 (2018).

41 Fagerberg, L. et al. Analysis of the human tissue-specific expression by genome-wide integration of transcriptomics and antibody-based proteomics. Molecular \& Cellular Proteomics 13, 397-406 (2014).

42 Kupershmidt, I. et al. Ontology-based meta-analysis of global collections of high-throughput public data. PLoS One 5, doi:10.1371/journal.pone.0013066 (2010).

43 Chattopadhyay, A. \& Ganapathiraju, M. K. Demonstration Study: A Protocol to Combine Online Tools and Databases for Identifying Potentially Repurposable Drugs. Data 2, 15 (2017).

44 Fridlender, Z. G. et al. Transcriptomic analysis comparing tumor-associated neutrophils with granulocytic myeloidderived suppressor cells and normal neutrophils. PloS one 7, e31524 (2012).

45 Kim, Y. et al. Major carcinogenic pathways identified by gene expression analysis of peritoneal mesotheliomas following chemical treatment in F344 rats. Toxicology and applied pharmacology 214, 144-151 (2006).

46 Blackshear, P. E. et al. Spontaneous mesotheliomas in F344/N rats are characterized by dysregulation of cellular growth and immune function pathways. Toxicologic pathology 42, 863-876 (2014).

47 Shukla, A. et al. Alterations in gene expression in human mesothelial cells correlate with mineral pathogenicity. American journal of respiratory cell and molecular biology 41, 114-123 (2009).

48 Dragon, J., Thompson, J., MacPherson, M. \& Shukla, A. Differential susceptibility of human pleural and peritoneal mesothelial cells to asbestos exposure. Journal of cellular biochemistry 116, 1540-1552 (2015).

49 Borczuk, A. et al. Molecular profiling of malignant peritoneal mesothelioma identifies the ubiquitin-proteasome pathway as a therapeutic target in poor prognosis tumors. Oncogene 26, 610-617 (2007).

50 Davidson, B. et al. Gene expression signatures differentiate ovarian/peritoneal serous carcinoma from diffuse malignant peritoneal mesothelioma. Clinical cancer research 12, 5944-5950 (2006).

51 Yanamala, N. et al. Characterization of pulmonary responses in mice to asbestos/asbestiform fibers using gene expression profiles. Journal of Toxicology and Environmental Health, Part A 81, 60-79 (2018).

52 Maxim, L. D. \& McConnell, E. A review of the toxicology and epidemiology of wollastonite. Inhalation Toxicology 17, 451-466 (2005).

Uhlen, M. et al. A pathology atlas of the human cancer transcriptome. Science 357, eaan2507 (2017). 
Piñero, J. et al. DisGeNET: a comprehensive platform integrating information on human disease-associated genes and variants. Nucleic acids research, gkw943 (2016).

55 Cacheiro, P., Haendel, M. A., Smedley, D., Consortium, I. M. P. \& Initiative, M. New models for human disease from the International Mouse Phenotyping Consortium. Mammalian Genome 30, 143-150 (2019).

56 Westbom, C. M. et al. CREB-induced inflammation is important for malignant mesothelioma growth. The American journal of pathology 184, 2816-2827 (2014).

57 Sirota, M. et al. Discovery and preclinical validation of drug indications using compendia of public gene expression data. Science translational medicine 3, 96ra77-96ra77 (2011).

58 Stahel, R., Weder, W., Lievens, Y. \& Felip, E. Malignant pleural mesothelioma: ESMO Clinical Practice Guidelines for diagnosis, treatment and follow-up. Annals of oncology 21, v126-v128 (2010).

59 Kindler, H. L. et al. Irinotecan for malignant mesothelioma: a phase II trial by the Cancer and Leukemia Group B. Lung Cancer 48, 423-428 (2005).

60 Fennell, D. A. et al. Efficacy and safety of first-or second-line irinotecan, cisplatin, and mitomycin in mesothelioma. Cancer 109, 93-99 (2007).

61 Han, B. et al. Combined use of irinotecan and p53 activator enhances growth inhibition of mesothelioma cells. FEBS Open bio 10, 2375-2387 (2020).

62 Koda, Y. et al. Irinotecan and Gemcitabine as Second-Line Treatment in Patients with Malignant Pleural Mesothelioma following Platinum plus Pemetrexed Chemotherapy: A Retrospective Study. Oncology, 1-8 (2020).

63 Choi, M. K. et al. Phase I study of intraperitoneal irinotecan in patients with gastric adenocarcinoma with peritoneal seeding. Cancer chemotherapy and pharmacology 67, 5-11 (2011).

64 Le, D. T., Deavers, M., Hunt, K., Malpica, A. \& Verschraegen, C. F. Cisplatin and irinotecan (CPT-11) for peritoneal mesothelioma. Cancer investigation 21, 682-689 (2003).

65 Van Meerbeeck, J. et al. Paclitaxel for malignant pleural mesothelioma: a phase II study of the EORTC Lung Cancer Cooperative Group. British journal of cancer 74, 961-963 (1996).

66 Vogelzang, N. et al. High-dose paclitaxel plus G-CSF for malignant mesothelioma: CALGB phase II study 9234. Annals of Oncology 10, 597-600 (1999).

67 Fizazi, K. et al. Combination raltitrexed (Tomudex ${ }^{\circledR}$ )-oxaliplatin: a step forward in the struggle against mesothelioma? The Institut Gustave Roussy experience with chemotherapy and chemo-immunotherapy in mesothelioma. European Journal of Cancer 36, 1514-1521 (2000).

69 Yamada, S. et al. Phase I/II study of adding intraperitoneal paclitaxel in patients with pancreatic cancer and peritoneal metastasis. The British journal of surgery 107, 1811 (2020). 

chemotherapy for advanced gastric cancer with serosal exposure. Case Reports in Oncology 7, 58-64 (2014).

71 Hartman, M.-L., Esposito, J. M., Yeap, B. Y. \& Sugarbaker, D. J. Combined treatment with cisplatin and sirolimus to enhance cell death in human mesothelioma. The Journal of thoracic and cardiovascular surgery 139, 1233-1240 (2010).

72 Stallone, G., Infante, B., Cormio, L., Macarini, L. \& Grandaliano, G. Rapamycin treatment for benign multicystic peritoneal mesothelioma: a rare disease with a difficult management. The American Journal of Case Reports 18, 627 (2017).

73 Xiang, S. et al. Rapamycin inhibits epithelial-to-mesenchymal transition of peritoneal mesothelium cells through regulation of Rho GTPases. The FEBS journal 283, 2309-2325 (2016).

74 Lee, J. J., Trizna, Z., Hsu, T., Spitz, M. R. \& Hong, W. K. A statistical analysis of the reliability and classification error in application of the mutagen sensitivity assay. Cancer Epidemiology and Prevention Biomarkers 5, 191-197 (1996).

75 Gultekin, K. et al. Effects of cisplatin and panobinostat on human mesothelial (Met-5A) and malignant pleural mesothelioma (MSTO-211H) cells. Genet Mol Res 12, 5405-5413 (2013).

76 Arrieta, O. et al. First-line chemotherapy with liposomal doxorubicin plus cisplatin for patients with advanced malignant pleural mesothelioma: phase II trial. British journal of cancer 106, 1027-1032 (2012).

77 Bertino, P. et al. Preliminary data suggestive of a novel translational approach to mesothelioma treatment: imatinib mesylate with gemcitabine or pemetrexed. Thorax 62, 690-695 (2007).

Tsao, A. S. et al. Phase I trial of cisplatin, pemetrexed, and imatinib mesylate in chemonaive patients with unresectable malignant pleural mesothelioma. Clinical lung cancer 15, 197-201 (2014).

79 Hunt, K. J., Longton, G., Williams, M. A. \& Livingston, R. B. Treatment of malignant mesothelioma with methotrexate and vinblastine, with or without platinum chemotherapy. Chest 109, 1239-1242 (1996).

80 Tsavaris, N. et al. Combination chemotherapy with cisplatin-vinblastine in malignant mesothelioma. Lung Cancer 11, 299-303 (1994).

81 Muers, M. et al. BTS randomised feasibility study of active symptom control with or without chemotherapy in malignant pleural mesothelioma: ISRCTN 54469112. Thorax 59, 144-148 (2004).

82 Kanellakis, N. I. et al. Patient-derived malignant pleural mesothelioma cell cultures: a tool to advance biomarkerdriven treatments. Thorax 75, 1004-1008 (2020).

83 Vogelzang, N. J. et al. Dihydro-5-azacytidine in malignant mesothelioma: A Phase II trial demonstrating activity accompanied by cardiac toxicity. Cancer: Interdisciplinary International Journal of the American Cancer Society 79, 2237-2242 (1997).

84 Hurwitz, J. L. et al. Vorinostat/SAHA-induced apoptosis in malignant mesothelioma is FLIP/caspase 8-dependent and HR23B-independent. European Journal of Cancer 48, 1096-1107 (2012). 
Krug, L. M. et al. Vorinostat in patients with advanced malignant pleural mesothelioma who have progressed on previous chemotherapy (VANTAGE-014): a phase 3, double-blind, randomised, placebo-controlled trial. The Lancet Oncology 16, 447-456 (2015).

Tanaka, M. et al. Inhibition of NADPH oxidase 4 induces apoptosis in malignant mesothelioma: Role of reactive oxygen species. Oncology reports 34, 1726-1732 (2015).

87 Demiroglu-Zergeroglu, A., Basara-Cigerim, B., Kilic, E. \& Yanikkaya-Demirel, G. The investigation of effects of quercetin and its combination with cisplatin on malignant mesothelioma cells in vitro. BioMed Research International 2010 (2010).

88 Lee, Y.-J. et al. Quercetin exerts preferential cytotoxic effects on malignant mesothelioma cells by inducing p53 expression, caspase-3 activation, and apoptosis. Molecular \& Cellular Toxicology 11, 295-305 (2015).

89 Kinoh, H. et al. Nanomedicines eradicating cancer stem-like cells in vivo by pH-triggered intracellular cooperative action of loaded drugs. ACS nano 10, 5643-5655 (2016).

90 Han, E. S. et al. Ruxolitinib synergistically enhances the anti-tumor activity of paclitaxel in human ovarian cancer. Oncotarget 9, 24304 (2018).

91 Steele, J. et al. Phase II trial of liposomal daunorubicin in malignant pleural mesothelioma. Annals of oncology 12, 497-499 (2001).

92 Fléchon, A., Lombard-Bohas, C., Boulez, J., Blay, J.-Y. \& Scoazec, J.-Y. Complete response of an HIV negative gastric Kaposi's sarcoma (KS) patient with peritoneal carcinomatosis by liposomal daunorubicin treatment. Annals of oncology 12, 275-276 (2001).

93 Kurashige, J. et al. Integrated molecular profiling of human gastric cancer identifies DDR2 as a potential regulator of peritoneal dissemination. Scientific reports 6, 22371 (2016).

94 Safra, T. et al. Weekly topotecan for recurrent ovarian, fallopian tube and primary peritoneal carcinoma: Tolerability and efficacy study-The Israeli experience. International Journal of Gynecologic Cancer 23 (2013).

95 Jang, Y.-H. et al. Effects of dexamethasone on the TGF- $\beta$ 1-induced epithelial-to-mesenchymal transition in human peritoneal mesothelial cells. Laboratory investigation 93, 194-206 (2013).

96 Liu, F. et al. Nintedanib inhibits the development and progression of peritoneal fibrosis. (2020).

97 Kuribayashi, K. et al. Methotrexate and gemcitabine combination chemotherapy for the treatment of malignant pleural mesothelioma. Molecular and clinical oncology 1, 639-642 (2013).

Solheim, $\varnothing$., Saeter, G., Finnanger, A. \& Stenwig, A. High-dose methotrexate in the treatment of malignant mesothelioma of the pleura. A phase II study. British journal of cancer 65, 956-960 (1992).

99 Yamao, T. et al. Phase II study of sequential methotrexate and 5-fluorouracil chemotherapy against peritoneally disseminated gastric cancer with malignant ascites: a report from the Gastrointestinal Oncology Study Group of the Japan Clinical Oncology Group, JCOG 9603 Trial. Japanese journal of clinical oncology 34, 316-322 (2004). 

pleural mesothelioma (MPM). Journal of Thoracic Oncology 10, 387-391 (2015).

101 Pignochino, Y. et al. The combination of sorafenib and everolimus shows antitumor activity in preclinical models of malignant pleural mesothelioma. BMC cancer 15, 1-13 (2015).

Duman, S. et al. in Advances in peritoneal dialysis. Conference on Peritoneal Dialysis. 104-110.

103 Batirel, S. et al. Resveratrol Inhibits Malign Pleural Mesothelioma Cell Proliferation Through Antioxidant System. Free Radical Biology and Medicine 86, S33-S34 (2015).

104 Lee, Y.-J. et al. Resveratrol contributes to chemosensitivity of malignant mesothelioma cells with activation of p53. Food and chemical toxicology 63, 153-160 (2014).

Mikuła-Pietrasik, J., Sosińska, P. \& Książek, K. Resveratrol inhibits ovarian cancer cell adhesion to peritoneal mesothelium in vitro by modulating the production of $\alpha 5 \beta 1$ integrins and hyaluronic acid. Gynecologic oncology 134, 624-630 (2014).

Liu, Z. \& Klominek, J. Regulation of matrix metalloprotease activity in malignant mesothelioma cell lines by growth factors. Thorax 58, 198-203 (2003).

lishi, H. et al. Genistein attenuates peritoneal metastasis of azoxymethane-induced intestinal adenocarcinomas in Wistar rats. International journal of cancer 86, 416-420 (2000).

van Breukelen, F. J. et al. Mitoxantrone in malignant pleural mesothelioma: a study by the EORTC Lung Cancer Cooperative Group. European Journal of Cancer and Clinical Oncology 27, 1627-1629 (1991).

Maiche, A., Virkkunen, P., Kontkanen, T., Möykkynen, K. \& Porkka, K. Bleomycin and mitoxantrone in the treatment of malignant pleural effusions. A comparative study. American journal of clinical oncology 16, 50-53 (1993).

110 Bairy, K. et al. Evaluation of intraperitoneal vincristine in malignant peritoneal effusion. Indian journal of physiology and pharmacology 47, 270-278 (2003).

111 Panou, V. et al. Frequency of germline mutations in cancer susceptibility genes in malignant mesothelioma. Journal of Clinical Oncology 36, 2863 (2018).

112 Barabási, A.-L., Gulbahce, N. \& Loscalzo, J. Network medicine: a network-based approach to human disease. Nature reviews genetics 12, 56-68 (2011).

113 Dey, A. et al. Loss of the tumor suppressor BAP1 causes myeloid transformation. Science 337, 1541-1546 (2012).

114 Shannon, P. et al. Cytoscape: a software environment for integrated models of biomolecular interaction networks. Genome research 13, 2498-2504 (2003).

115 Consortium, G. O. The Gene Ontology (GO) database and informatics resource. Nucleic acids research 32, D258D261 (2004). 
117 Jain, A. \& Tuteja, G. TissueEnrich: Tissue-specific gene enrichment analysis. Bioinformatics 35, 1966-1967, doi:10.1093/bioinformatics/bty890 (2019).

118 Wishart, D. S. et al. DrugBank: a comprehensive resource for in silico drug discovery and exploration. Nucleic acids research 34, D668-D672 (2006). 


\section{Supplementary Files}

This is a list of supplementary files associated with this preprint. Click to download.

- CombinedSupplementaryFilesv1.xlsx 